\title{
Divergent effects of oxytocin on eye contact in bonobos and chimpanzees
}

\section{$\operatorname{AUTHOR}(\mathrm{S})$ :}

Brooks, James; Kano, Fumihiro; Sato, Yutaro; Yeow, Hanling; Morimura, Naruki; Nagasawa, Miho; Kikusui, Takefumi; Yamamoto, Shinya

\section{CITATION:}

Brooks, James ...[et al]. Divergent effects of oxytocin on eye contact in bonobos and chimpanzees. Psychoneuroendocrinology 2021, 125: 105119.

\section{ISSUE DATE:}

2021-03

URL:

http://hdl.handle.net/2433/260851

\section{RIGHT:}

(c) 2021. This manuscript version is made available under the CC-BY-NC-ND 4.0 license

http://creativecommons.org/licenses/by-nc-nd/4.0/:; The full-text file will be made open to the public on 1 March 2022 in accordance with publisher's 'Terms and Conditions for Self-Archiving'.; This is not the published version. Please cite only the published version.; この論文は出版社版でありません。引用の際には出版社版をご確認ご利用ください。 


\section{Divergent effects of oxytocin on eye contact in bonobos and chimpanzees}

2

3 James Brooks ${ }^{1,2} *$, Fumihiro Kano ${ }^{1,2} *$, Yutaro Sato ${ }^{1,2}$, Hanling Yeow ${ }^{1,2}$, Naruki Morimura ${ }^{1,2}$, Miho

$4 \quad$ Nagasawa $^{3}$, Takefumi Kikusui ${ }^{3}$, Shinya Yamamoto ${ }^{1,4}$

$5 \quad{ }^{1}$ Wildlife Research Center, Kyoto University

$6{ }^{2}$ Kumamoto Sanctuary, Kyoto University

$7 \quad{ }^{3}$ School of Veterinary Medicine, Azabu University

$8{ }^{4}$ Institute for Advanced Study, Kyoto University

9

*These authors contributed equally to this work

To whom correspondence should be addressed.

Sakyo, Kyoto, 606-8203, Japan 
18

19

20

21

22

\section{Abstract}

Oxytocin has drawn significant research attention for its role in modulating mammalian social behavior. Despite generally conserved roles, oxytocin can function differently even in closely related species. Previous studies have shown that bonobos and chimpanzees, humans' two closest relatives, demonstrate considerable behavioral differences, including that bonobos look more at others' eyes than chimpanzees. Oxytocin is known to increase attention to another's eyes in many mammalian species (e.g. dogs, monkeys, and humans), yet this effect has not been tested in any nonhuman great ape species. This study examined how intranasally-administered oxytocin affects eye contact in bonobos and chimpanzees using eye tracking. Following administration of either oxytocin or saline control with a nebulizer, chimpanzees $(n=6)$ and bonobos $(n=5)$ were shown images of conspecific faces while their eye movement was recorded. Oxytocin changed the eyelooking behavior of bonobos and chimpanzees differently. We found that oxytocin increased eye contact in bonobos but not chimpanzees; while one chimpanzee showed an increase, interestingly, 5 out of 6 chimpanzees showed decreased looking to the eyes compared to the mouth, suggesting moderate eye avoidance. Given the importance of eye contact in their social interactions, our results suggest that oxytocin may play modulatory roles in bonobos' and chimpanzees' species-specific social behavior and underscore the importance of oxytocin in hominid social evolution.

Keywords: Oxytocin, eye contact, bonobos, chimpanzees, species differences, social attention 


\section{Introduction}

Oxytocin (OT) has gained growing research interest in past decades due to its diverse regulatory roles in mammalian social behaviors. The function of OT is largely conserved across mammals, specifically that it regulates essential reproductive needs, such as maternal attachment and pair bonding (Anacker and Beery, 2013), as well as nuanced socio-cognitive behavior and cognition, such as non-kin social bonding (Crockford et al., 2013; Romero et al., 2014; Wittig et al., 2014), outgroup mentality (De Dreu et al., 2010; Samuni et al., 2017), social attention (Dal Monte et al., 2014; Guastella et al., 2008), and empathy (Burkett et al., 2016). However, despite its largely conserved roles, previous studies have demonstrated that the role of OT on behavior differs even between closely related mammalian species, partly due to differential distributions of OT receptors in their brains (Anacker and Beery, 2013; Insel and Shapiro, 1992). Studies have also demonstrated that genes encoding the receptors of OT (OXTR) and the structurally similar neuropeptide arginine vasopressin (AVP) can change rapidly in evolution (Hammock and Young, 2005), and that polymorphisms in these genes are related to the expression of social behaviors across individuals and species (Hopkins et al., 2012; Rodrigues et al., 2009; Staes et al., 2014; Staes et al., 2016).

These pieces of evidence offer a potential mechanism by which species-typical social behaviors can rapidly evolve in closely related mammalian species (Insel and Young, 2000). In a similar vein, some researchers suggest that animal domestication is facilitated by changes in the OT and AVP systems and the associated reduction of stress sensitivity and aggression (Herbeck and Gulevich, 2019) - thought to be essential changes for nonhuman animals integrating into a human society.

Given this accumulating evidence, researchers also suspect that neuropeptides may have played a key role in the evolution of species-typical behaviors in bonobos and chimpanzees, humans' two closest relatives (Staes et al., 2014). Bonobos and chimpanzees diverged only recently 
in evolution, ( 1-2 million years ago; Prufer et al., 2012), but differ in social organization and a number of important social behaviors (Hare and Yamamoto, 2017). Critically, bonobos and chimpanzees differ in tolerance and aggression, particularly to outgroup individuals (Samuni et al., 2017; Tan and Hare, 2013; Tokuyama et al., 2019), socio-sexual behaviors (De Waal, 1990b) and social attention (Herrmann et al., 2010; Kano et al., 2015), all of which are known to be regulated by OT in human and nonhuman mammals (Anacker and Beery, 2013; Bartz et al., 2011; Bauman et al., 2018). Moreover, bonobos and chimpanzees differ neuroanatomically in brain areas related to socio-emotional behavior, such as the amygdala (and its connection to the anterior cingulate cortex) and insular cortex (Hopkins et al., 2015; Issa et al., 2019; Rilling et al., 2012; Staes et al., 2018; Stimpson et al., 2016), which are known to be modulated by OT (Burkett et al., 2016; Gamer et al., 2010; Rogers-Carter et al., 2018). Studies have also shown that bonobos and chimpanzees differ in OT and AVP receptor genes (Staes et al., 2014), that polymorphisms in these genes are linked with personality in bonobos and chimpanzees (Anestis et al., 2014; Hopkins et al., 2012; Staes et al., 2016; Wilson et al., 2017), and that urinary oxytocin increases after engaging in species-typical behavior, such as non-copulatory sex in bonobos (Moscovice et al., 2019) and intergroup conflict in chimpanzees (Samuni et al., 2017). One theory proposes that bonobos may have undergone a domestication-like process which may have reduced aggression in their evolution (Hare et al., 2012), a process which might be related to the changes in OT/AVP systems (Herbeck and Gulevich, 2019). Therefore, these studies suggest that species-typical behaviors of bonobos and chimpanzees may have coevolved with the OT/AVP system. However, despite this accumulating evidence, no comparative study has been conducted to test whether OT affects the behavior of these species differently under the same experimental conditions. To this end, intranasal administration of OT seems most effective. 
Several previous studies have demonstrated that intranasal administration of OT causes diverse effects on behavior and cognition in human and nonhuman primates. In studies with humans, OT has been found to be associated with alleviation of social anxiety (Macdonald and Macdonald, 2010), enhancement of prosocial behavior such as increased trust and donation (e.g. Israel et al., 2009; Kosfeld et al., 2005), and enhancement of attention to certain social stimuli such as eyes (Andari et al., 2010; Auyeung et al., 2015; Guastella et al., 2008). OT has also been found to be associated with derogation of the outgroup (De Dreu et al., 2010), enhancement of negative emotion such as envy and schadenfreude (Shamay-Tsoory et al., 2009), and reduced avoidance of negative (non-social) stimuli (Harari-Dahan and Bernstein, 2017). These studies and others have stressed multifunctionality of OT, namely that OT has both prosocial and antisocial as well as social and non-social effects, which has given rise to the social-salience (Shamay-Tsoory and Abu-Akel, 2016), social approach-withdrawal (Kemp and Guastella, 2011), and general approach-avoidance hypotheses (Harari-Dahan and Bernstein, 2014). In studies with macaques, the effect of OT on social orientation and attention is well studied. In addition to enhancing social proximity (Simpson et al., 2014), nebulized intranasal OT increases attention to eye gaze (Dal Monte et al., 2014; Ebitz et al., 2013; also see Kotani et al., 2017 with marmosets), enhances gaze-following (Putnam et al., 2016), and attenuates attention to both negative facial expressions (Parr et al., 2013) and threat staring to others (Jiang and Platt, 2018a). One study also found antisocial OT effects (e.g. increase in threat staring to males) in female macaques following OT administration (Jiang and Platt, 2018b), highlighting the complex effects of OT depending on subjects' biological differences and social contexts.

There have been fewer studies about the oxytocinergic system in non-human great apes and no studies investigating its effect on social attention, though field studies measuring urinary OT suggest an important role of OT in several key social behaviors. In addition to the previously 
mentioned studies reporting increased urinary OT following non-copulatory sex in bonobos (Moscovice et al., 2019) and intergroup encounters in chimpanzees (Samuni et al., 2017), urinary OT in wild chimpanzees has been found to rise following food sharing (Wittig et al., 2014), grooming (Crockford et al., 2013), group hunting (Samuni et al., 2018) and reconciliation (Preis et al., 2018). Two previous studies (Hall et al., 2019; Proctor et al., 2016) have administered nebulized OT to chimpanzees to test its effects on their real-life social interaction. In particular, in Proctor et al. (2016), one individual was administered OT or placebo, and was then observed for their daily social interaction with individuals who did not receive OT, though they found no statistically significant differences between conditions. In Hall et al. (2019), no consistent patterns in a token exchange task were found with or without OT administration. As authors noted, both studies yielded null results presumably due to several methodological limitations; for example, in optimizing OT administration procedures for great ape species or in detecting subtle changes in social behaviors in complex interactions with non-OT-administered individuals.

This study tested whether intranasal administration of nebulized OT affects eye contact behavior in bonobos and chimpanzees similarly or differently using an eye tracking setup. Eye contact plays significant roles in primate cognition and social communication (Emery, 2000) and the function of eye contact is largely similar among primate species, including bonobos and chimpanzees. For example, staring at another's eyes signals threat (Emery, 2000) as well as affiliation (e.g. mutual gaze between mothers and infants Ferrari et al., 2009). Chimpanzees establish eye contact before attempting to reconcile with others after fights (De Waal, 1990a). Bonobos engage in prolonged eye contact during characteristic non-copulatory sex (GG-rubbing; Annicchiarico et al., 2020; Moscovice et al., 2019). On the other hand, however, bonobos and chimpanzees seem to differ significantly in their sensitivity to others' gaze (as among other closely related primates; Thomsen, 1974). Specifically, in eye tracking tests, bonobos viewed the eyes of 
conspecific facial images longer than chimpanzees (Kano et al., 2015). This same pattern is also observed in response to a human experimenter's eyes (Mulholland et al., 2020). Relatedly, in a battery of tests examining cognitive differences between bonobos and chimpanzees, bonobos followed the experimenter's gaze more frequently than chimpanzees (Herrmann et al., 2010). This study thus asked whether OT modulates the species differences in eye contact behavior in bonobos and chimpanzees.

We predicted that the use of an eye tracking setup may reveal subtle changes in the effect of OT on eye movement in bonobos and chimpanzees. Regarding the direction of change, our first hypothesis was that OT administration would increase eye contact in both species, consistent with the previous findings with both neurotypical and autistic humans (Andari et al., 2010; Auyeung et al., 2015; Gamer et al., 2010; Guastella et al., 2008), macaques (Dal Monte et al., 2014; Ebitz et al., 2013), marmosets (Kotani et al., 2017), and dogs (Nagasawa et al., 2015). Thus, administration of OT may alter chimpanzees' looking pattern to become more like that of bonobos by increasing attention to the eye region. Our second hypothesis was that OT administration would differently affect eye contact in bonobos and chimpanzees, consistent with the findings that bonobos and chimpanzees differ in neural and genetic structures related to OT receptor systems (Hopkins et al., 2015; Issa et al., 2019; Rilling et al., 2012; Staes et al., 2018; Staes et al., 2014; Stimpson et al., 2016). Given that bonobos are more sensitive to others' eyes than chimpanzees, OT may enhance such pre-existing eye sensitivity, particularly in bonobos but not chimpanzees.

\section{Material and methods}

\subsection{Participants.}


Six chimpanzees (Pan troglodytes verus) and five bonobos (Pan paniscus) living at Kumamoto Sanctuary, Japan, participated in this study (Table 1). One additional bonobo male was tested but excluded from the analyses because he rejected inhaling nebulized mist.

\subsection{Ethics statements.}

All ape participants were tested in rooms prepared for each species, and their daily participation in this study was voluntary. They received regular feedings, daily enrichment, and had ad libitum access to water. No changes were made to their daily care routine. Research protocol was approved by the institutional review board (WRC-2019-KS013A for chimpanzees, and WRC-2019-KS014A for bonobos). Safety of the OT administration was carefully considered and accepted given the fact that 1) OT is often administered to human children and adults, that 2) OT is active for only a short period of time following administration with no known side effects in humans (MacDonald et al., 2011), that 3) OT is naturally produced in bonobos and chimpanzees following relevant behaviors (Crockford et al., 2013; Moscovice et al., 2019), and that 4) previous two studies administering OT intranasally to chimpanzees did not report any agonistic interaction (Hall et al., 2019; Proctor et al., 2016). In addition, we conducted a pilot test with chimpanzees following the same OT administration procedure and confirmed no irregular behaviors or interactions were observed.

\subsection{Oxytocin administration apparatus and procedure.}

We modelled our general OT administration procedure on methods commonly adopted in tests with macaques (for a review, see Bauman et al., 2018). In particular, we did not use nasal spray but instead used a nebulizer to administer aerosolized OT to ape participants. This procedure has proven to be effective in a number of behavioral and physiological tests with macaques, including the observation that OT level in cerebrospinal fluid (CSF) increased following administration of 
174

aerosolized OT (Modi et al., 2014). OT or saline placebo were administered with a portable nebulizer (NE-U22-4, Omron, Kyoto, Japan) to apes in a custom-designed box while apes were drinking a dripping of juice thorough a nozzle attached to the box $(13 \mathrm{w} \times 16.5 \mathrm{~h} \times 8 \mathrm{~d} \mathrm{~cm})$, via a custom-made juice dispenser (Figure 1A; similar to the device used and validated by Parr et al., 2013). Criteria for successful administration was 4 cumulative minutes, counted with a stopwatch, of nebulized mist being projected onto their nose inside the box. We paused counting when the nose of ape was out of the box. All apes (except one bonobo who dropped out) completed this procedure within 10 minutes in each trial. We used a concentration of $40 \mathrm{IU} / \mathrm{mL}$ of oxytocin, which was nebulized at a rate of $0.25 \mathrm{ml} /$ minute, meaning roughly $40 \mathrm{IU}$ of oxytocin was nebulized in the cumulative 4 minutes. A dose of 40 IU was chosen because it is well within the range of human and monkey studies (commonly 24-40 IU; Bauman et al., 2018; MacDonald et al., 2011), and a relatively high dose was chosen because certain amount of the mist was expected to evaporate from the box (as in Parr et al., 2013). We visually confirmed that individuals breathed the mist through their nose during administration. The eye tracking test on each day was both started and completed 30-60 minutes after the end of administration procedure, an interval also well within that of previous studies (Bauman et al., 2018).

\subsection{Eye tracking apparatus.}

Following an established procedure (Kano et al., 2011; Krupenye et al., 2016), ape eyes were recorded by an infrared head-free eye tracker $(300 \mathrm{~Hz}, \mathrm{TX} 300$, Tobii Technology AB). Apes sipped a dripping of juice via a custom-made juice dispenser while they viewed the stimuli. The stimuli were presented with a resolution of $1,280 \times 720$ pixels at a viewing distance of $70 \mathrm{~cm}$ on a 23 -inch LCD monitor $\left(43 \times 24^{\circ}\right)$ with Tobii Studio software (ver. 3.2.1). Due to apes' relatively short attention span, automated calibration was conducted at two points for each ape by presenting a 
small object or video clip on each reference point. Subsequently, we checked the quality of calibrations by presenting small reference icons on the monitor before each recording session and confirming the ape's gaze did not deviate from the icons. We repeated the calibration procedure whenever necessary. Following these procedures, calibration errors in apes are typically within one degree (Kano et al., 2011), an accuracy sufficient to distinguish between eyes and mouth of presented faces in this study.

\subsection{Stimuli and procedure.}

Stimuli consisted of two 3-minute videos for each species including both movies and slideshows of images. Each image was of conspecific faces and was presented for three seconds each, and there were approximately 30 images in each video. To maximize each species' interest and natural eye movement responses to the faces, we presented complex scenes depicting various natural behaviors (resting, fighting, playing, copulating, displaying, grooming, and tool-using), and faces with varying facial expression (neutral, play, and scream faces, and grimaces), gaze direction (direct and averted), and posture, of individuals of all ages and sexes, and of both ingroup and outgroups (see Supplementary Excel file for more details). Both species viewed only conspecifics (images containing themself were excluded from the analysis). Some individuals appeared in multiple images (maximally in 4 images) with different camera angles and configurations. The contents and configurations of scenes were matched as much as possible between the videos prepared for each species (Figure 1B). Two different videos of 3 minutes each were prepared for each species (Chimpanzee/Bonobo Video 1 and 2) which each individual saw twice (across conditions).

Following administration of either OT or saline, each ape saw one video each day (this is a trial).

The order of OT and saline administration (Condition) was counterbalanced across individuals, either in ABBA or BAAB order. The first two trials (Block 1) presented Video 1 and latter two 
trials (Block 2) presented Video 2. Thus, each ape saw each of two videos twice on consecutive trials (this is a block). There were thus four trials for each ape. Each trial was separated by a minimum of 5 days to avoid any possible lasting effects of OT.

\subsection{Analysis.}

Eye movement was filtered using Tobii Fixation Filter with default parameters. Areas-OfInterest (AOIs) were defined for each cut of movies and each picture in slideshows in the Tobii Studio software (see Figure S1 for examples). AOIs included the eyes, mouth, face (including both eyes and mouth), genital, body (including face and genital), and action target (object, food, and tool held by the hands). In each image, AOI was not defined if its minimal diameter was smaller than one degree (e.g. the vertical diameter of the eye AOI), or the target object was moving rapidly or covered (consequently, eye/mouth AOI was removed in movies). Eye and mouth AOIs were defined as a pair in all pictures.

Statistical analyses were performed in R using linear mixed models (LMM) ('lmer' in the package ' $1 m e 4$ ') with Gaussian error structure and identity link function. The dependent variables were total looking duration to each AOI in each trial (presenting a 3-min video); eyes, mouth, face (as a whole), genital, action targets, body (as a whole), and screen (i.e. a whole video screen) and separate models were run for each of these dependent variables. The variation in presentation durations of each AOI across videos was minimal for the eye, mouth, and face AOIs (and absent for the body and screen AOIs), but relatively large for target and genital AOIs (Table S1). Thus, to analyze the looking duration to the latter two AOIs, we used the proportion of looking duration (with respect to the total presentation duration of AOI) as the dependent variable (logit-transformed; Warton and Hui, 2011); for the looking duration to the former three AOIs, such a transformation was not necessary because the variation in presentation duration across videos was minimal, and we 
confirmed that the same results emerged with or without the transformation. We additionally tested the difference score subtracting eye looking duration from mouth looking duration in a post-hoc model (detailed in Result). In all models, we included Species (bonobos/chimpanzees), Condition (OT/saline), and interaction between the two factors as test predictors. Additionally, we included Block (1/2) and Trial (1/2), which was nested in Block, as control variables. These control variables were standardized (using the 'scale' function) according to the recommendation of Schielzeth (2010). We included participant and video as random intercepts and random slopes of all fixed effects; the random-effects structure was kept maximal to save conservativity of the tests according to the recommendation of Barr et al. (2013), except that we removed the correlation between the intercept (Participant) and slopes (Block/Trial) to keep sufficient random-effect variations. The model syntax in R (used for all models reported in the main texts, including the eye-minus-mouth model) was; Looking duration $\sim$ Species*Condition + Block/Trial $+(1+$ Condition + Block/Trial $\|$ Participant). The number of observations was 44 in this model (11 participants in 4 trials). We confirmed the assumptions of normally distributed and homogeneous residuals by visual inspection of diagnostic plots (q-q plots and scatterplots of the residuals plotted against fitted values) in all models. We also checked Variance Inflation Factors (VIF) in a R package 'car' and found that collinearity was not an issue in any model (all VIF $<3$ ). To check the model stabilities, we excluded each level of random effects (subject and video) one by one and calculated Cook's distances as measures of influence in a R package 'influence.Me'. When this manipulation suggested any influential cases (Cook's distance $>1$ ), we confirmed that excluding that influential case did not change the main results. We used a likelihood ratio test to examine the significance of an effect in question (using the 'drop1' function). We first tested the interaction term in each model, and if not significant, and then reran the model without the interaction term (Engqvist, 2005). When we found a significant interaction effect in the model, we further examined it by testing simple effects in the 

Material. caregivers and conspecific peers.

\begin{tabular}{lcccc}
\hline Species & Sex & Age & Rearing history & Name \\
bonobo & F & 29 & Nursery-peer & Ikela \\
bonobo & F & 38 & Mother & Lenore \\
bonobo & F & 48 & Nursery-peer & Louise \\
bonobo & F & 31 & Nursery-peer & Lolita \\
bonobo & M & 16 & Nursery-peer & Vijay \\
chimpanzee & F & 12 & Nursery-peer & Hatsuka \\
chimpanzee & F & 12 & Mother & Iroha \\
chimpanzee & M & 25 & Mother & Zamba \\
chimpanzee & F & 21 & Mother & Misaki \\
chimpanzee & F & 24 & Nursery-peer & Mizuki \\
chimpanzee & F & 15 & Mother & Natsuki \\
*For further information of these apes, visit GAIN (https:/shigen.nig.ac.jp/gain/; Great Ape \\
Information Network; the online studbook of Japanese apes) and type the names in the search bar.
\end{tabular}

subsets of data including each level of predictors. Finally, to explore whether any of the stimulus properties (e.g. gaze direction, ingroup/outgroup, male/female, facial expressions of the presented faces; see Supplementary Excel file) critically affected the results (eye-mouth difference looking score), we examined the effect of each stimulus property individually (for simplicity, the interaction between stimulus properties was not considered in this analysis) by restructuring the dataset to include three factors, species, condition, and stimulus property, and then testing the three-way interaction effect; the results from these additional analyses were reported in Supplemental

Table 1. Participant information. In the rearing history column, 'Mother' indicates the individuals reared by their biological mothers, and 'Nursery-peer' indicates the individuals reared by human 
Brooks et al. 14

A

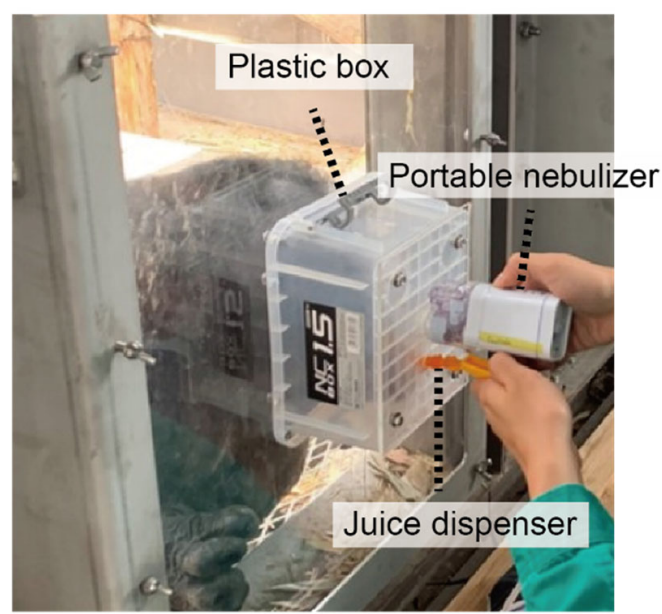

B

Bonobo stimuli

281

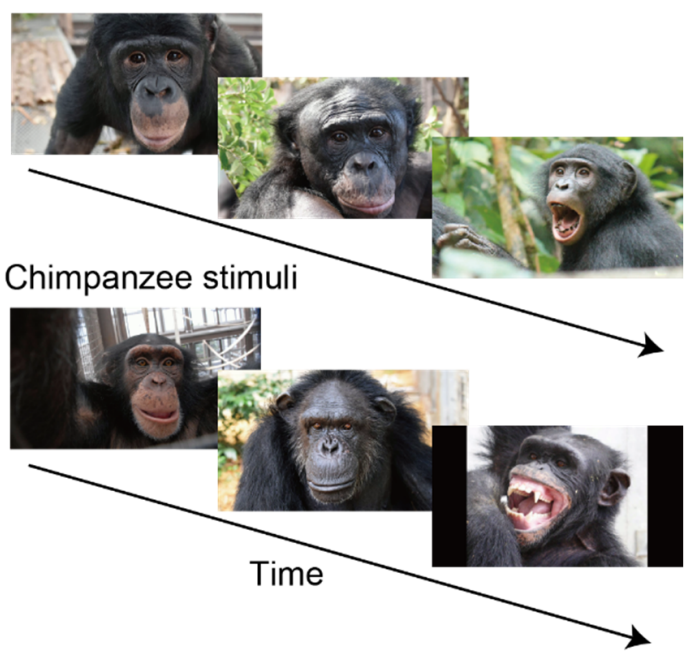

282 Figure 1: Study Design. (A) Oxytocin (OT) administration apparatus. (B) Examples of presented

283 images. Also see the link (https://youtu.be/ LbQ3qtlEcA) for more examples and superimposed eye movements of bonobos and chimpanzees. 


\section{Results}

OT differently affected bonobos' and chimpanzees' looking duration to the eye of the images (Figure 2A; a significant interaction effect between species and condition: $\beta=-3.4, \mathrm{SE}=1.4$, $\left.\mathrm{CI}_{\text {lower }}=-6.2, \mathrm{CI}_{\text {upper }}=-0.4, \chi^{2}=4.9, \mathrm{p}=0.028\right)$. OT did not significantly affect their looking duration to the other AOIs (see Table S2 for full statistical results). Although not significant, OT tended to affect differently the two species' looking duration to the mouth of the images (Figure 2B: $\beta=3.6, \mathrm{SE}=1.9, \mathrm{CI}_{\text {lower }}=-0.09, \mathrm{CI}_{\text {upper }}=7.5, \chi^{2}=3.2, \mathrm{p}=0.072$ ); the directions of these OT effects were the opposite of those on their responses to the eye of the images. As the responses to the eyes and mouth are essentially related (viewing mouth is not viewing eyes of the same face, and vice versa), and the two species did not differ in their overall looking duration to the faces of images (Table S2), we additionally ran a model with a difference score which subtracted looking duration to the mouth from that to the eyes in each image (Figure 2C). There was a significant interaction between species and condition $\left(\beta=-6.9, \mathrm{SE}=2.4, \mathrm{CI}_{\mathrm{lower}}=\right.$ 12.0, $\left.\mathrm{CI}_{\text {upper }}=-2.6, \chi^{2}=6.4, \mathrm{p}=0.011\right)$. To explore this observed interaction effect further, we tested simple effects in the subsets of data on each level of test predictors. We found that OT increased bonobos' looking duration to the eyes compared to the mouth $(\beta=3.5, \mathrm{SE}=0.9$, $\left.\mathrm{CI}_{\text {lower }}=1.8, \mathrm{CI}_{\text {upper }}=5.3, \chi^{2}=8.2, \mathrm{p}=0.004\right)$. On the other hand, OT did not significantly change chimpanzees' looking durations for the eyes compared to the mouth $(\beta=-3.5, \mathrm{SE}=2.6$, $\left.\mathrm{CI}_{\text {lower }}=-9.1, \mathrm{CI}_{\text {upper }}=1.6, \chi^{2}=1.5, \mathrm{p}=0.22\right)$. In neither condition did bonobos and chimpanzees differ significantly in their looking durations for the eyes compared to the mouth $\left(\mathrm{OT}: \beta=-9.0, \mathrm{SE}=7.3, \mathrm{CI}_{\text {lower }}=-23.1, \mathrm{CI}_{\text {upper }}=5.5, \chi^{2}=1.4, \mathrm{p}=0.24 ;\right.$ Saline: $\beta=-2.2, \mathrm{SE}=$ 6.4, $\mathrm{CI}_{\text {lower }}=-13.7, \mathrm{CI}_{\text {upper }}=9.8, \chi^{2}=0.1, \mathrm{p}=0.73$ ). Figure $2 \mathrm{C}$ show that the effect of OT was relatively consistent across individuals in bonobos. One chimpanzee (Iroha) showed the same direction of change after administration of OT as bonobos did, while the other five chimpanzees 
showed the opposite direction of change. Figure $2 \mathrm{C}$ also showed relatively large individual differences within each species in the eye-mouth looking duration, which explained the absence of a significant effect of species in this study. Finally, an inclusion of each stimulus property (group affiliation, sex, gaze direction, and facial expression of the presented faces) as an additional test predictor into the same model (with the eye-mouth difference score as the response) confirmed that the same interaction effect (species*condition) can be observed in these models. We additionally found the main effects of group affiliation and facial expression of the presented faces (see Figure S3 and Supplemental Results). Notably, we found a threeway interaction effect between species, condition, and gaze direction of faces. This result indicated that the species difference in OT effect can be observed when the two species viewed the faces with direct gaze, but not those with averted gaze, suggesting that OT affected the two species' eye contact behavior (i.e. looks to others' direct gaze). 
A

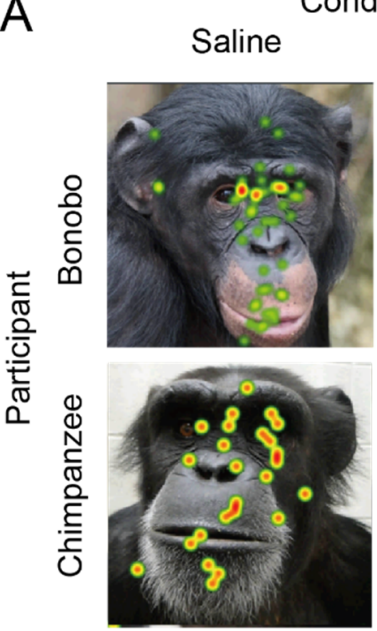

Condition Oxytocin

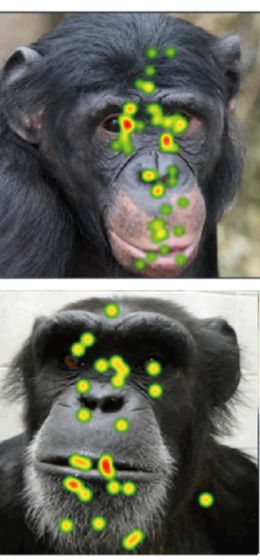

B

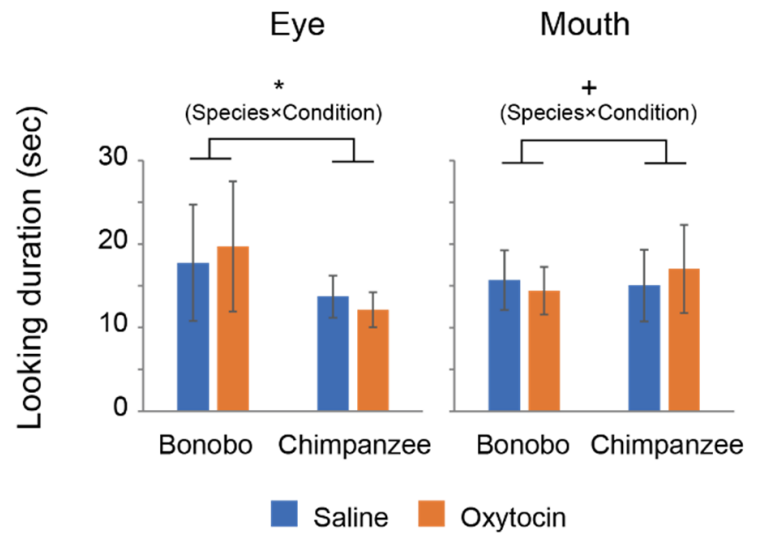

C
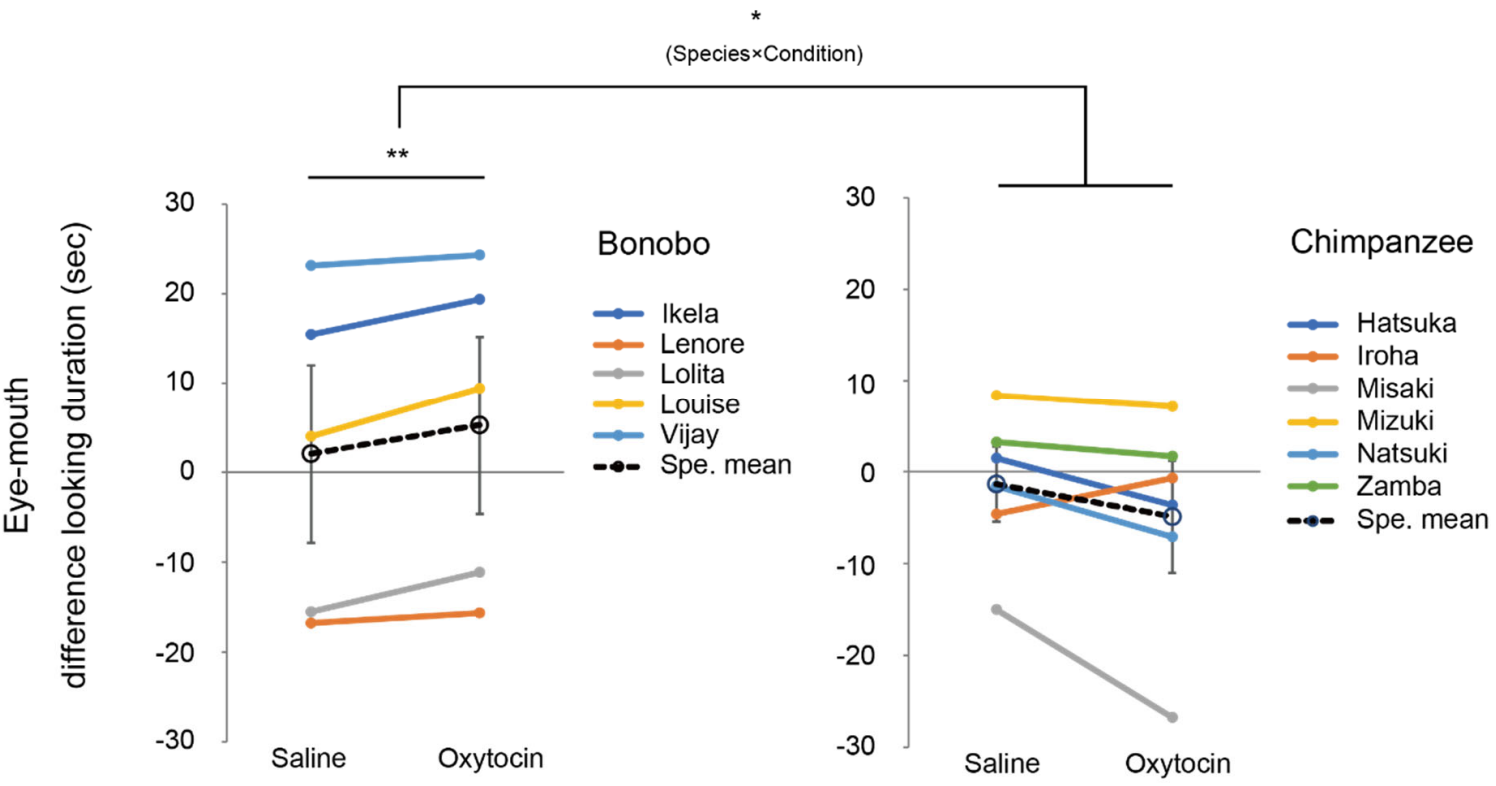

Figure 2: Effect of oxytocin on eye contact in bonobos and chimpanzees. (A) Heatmaps of eye fixations (redder parts indicate greater attention). (B) Mean looking durations (sec) for the eyes and mouth of faces in a trial (presenting a 3-min video). (C) Mean difference looking durations (sec) for the eyes and mouth (eyes minus mouth) by individuals (chimpanzees: $\mathrm{n}=6$, bonobos: $\mathrm{n}=5$, in 
329 solid lines) and also by each species (in dashed lines) in a trial (presenting a 3-min video). Error

330 bars denote $95 \%$ confidence intervals. ${ }^{* *} \mathrm{p}<0.01,{ }^{*} \mathrm{p}<0.05,+\mathrm{p}<0.1$. 


\section{Discussion}

Intranasal administration of nebulized OT increased looking duration to eyes of conspecific images in bonobos but not chimpanzees, supporting our second hypothesis predicting differential effects of OT on the eye looking behavior of these species. The increased eye looking observed in bonobos was consistent with a number of previous findings with human and nonhuman primates (Andari et al., 2010; Auyeung et al., 2015; Dal Monte et al., 2014; Ebitz et al., 2013; Gamer et al., 2010; Guastella et al., 2008; Kotani et al., 2017) and also dogs (Nagasawa et al., 2015). OT, however, did not affect chimpanzees in the same way. Interestingly, while one chimpanzee (Iroha) showed the same direction of change as bonobos did, 5 out of 6 chimpanzees decreased total looking duration at eyes relative to mouth. Most likely, looking at the mouth in chimpanzees indicates a moderate eye avoidance; that is, although they attended to faces (like bonobos), they did not look at eyes directly but looked slightly down (i.e. mouth). Thus, OT may not only operate differently but enhance species-typical behavioral tendencies in these species.

\section{Before proceeding to more detailed discussions about the hypotheses, several low-level} explanations can be ruled out. First, it is unlikely that procedural differences between species caused the differential OT effects on their behavior because we tested the two species using identical experimental apparatus and procedures. Second, it is unlikely that the dose of OT (40 IU) used in this study was inappropriate to test either species of great ape because this dose was well within the range of previous studies with macaques and humans of respectively smaller and larger body weights compared to bonobos/chimpanzees (Bauman et al., 2018; MacDonald et al., 2011), and we visually confirmed that individuals from both species breathed the mist through their nose during administration. Third, it is unlikely that the observed species difference is explained by a general increase in attention to the face because OT did not change overall attention to faces in either species. Moreover, we found that the observed species difference was particularly evident 
when the two species were viewing faces with direct gaze, but not with averted gaze. These results thus suggested that the observed OT effects reflect differential operation of OT on eye contact behavior in bonobos and chimpanzees.

Psychologically, among several hypotheses proposed in literature, the social salience hypothesis seems most parsimonious with our results. This hypothesis was proposed to explain both prosocial and antisocial effects of OT on human social behavior, and assumes that OT enhances pre-existing social sensitivities and ongoing psychological processes under given contexts (ShamayTsoory and Abu-Akel, 2016). In this study, therefore, OT may have enhanced pre-existing gaze sensitivity in bonobos. Other hypotheses, such as affiliative/prosocial and anxiety/stress-reduction hypotheses (Ebitz et al., 2013; Macdonald and Macdonald, 2010) may explain the results observed in bonobos (i.e. increase in eye contact) if attending to others' eyes is driven by affiliative motivation or is somewhat stressful (and then this stress was alleviated) in this species. However, these hypotheses do not explain why the same effects were not observed in chimpanzees. Another hypothesis, social approach-withdrawal hypothesis seems to fit our results relatively well because it predicts that OT facilitates the approach behavior of participants (Kemp and Guastella, 2011). Previously, it has been shown that the degree of eye contact between individuals varies as a function of both physical and psychological distances between individuals in both human and nonhuman primates (Argyle and Dean, 1965; Thomsen, 1974). In addition, social distances among individuals between bonobos and chimpanzees seem to differ by default, specifically that bonobos (especially females) are overall more gregarious than chimpanzees (Tokuyama et al., 2019).

Therefore, if OT facilitated social approach in both species, bonobos may have perceived the social stimuli as more relevant, leading to an increase in eye contact, while chimpanzees may not; in fact, some of them may have perceived the social stimuli as more stressful or threatening, leading to a decrease in eye contact. Future research should directly test these hypotheses, as well as examine 
whether the effect of OT is modulated by social distance. In any case, the most relevant point here is that OT may have acted differently in bonobos and chimpanzees by interacting with certain preexisting tendencies that each species had (or did not have).

Neurologically, the differential operation of OT on the two species is likely caused by differential distribution of OT receptors in brain (or differential density of OT receptors in particular brain regions) between species, as suggested in previous studies (Anacker and Beery, 2013; Insel and Shapiro, 1992). Unfortunately, much remains to be studied as to the neuroanatomical distribution/molecular structure of OT (and AVP) receptors in bonobos and chimpanzees. However, one study comparing OT- and AVP-synthesizing neurons in the hypothalamus did not find species differences between bonobos and chimpanzees (Hopkins et al., 2015), suggesting that the production of OT does not differ between the species (but the distribution, density, and binding affinity of OT receptors may). Our results thus encourage a comparative study of OT receptors in brain regions related to eye contact and social attention (e.g. amygdala), which likely differ between the species. To speculate the candidate region, a high-resolution fMRI study with humans (Gamer et al., 2010) may be particularly informative, which found that different subregions of the amygdala mediate valence-related and attention-related effects of OT. Of particular relevance to this study, an increase in activation of the posterior amygdala (likely basal nucleus) and a functional coupling of this region to the superior colliculi was observed in response to the eyes of presented faces in the OT compared to placebo condition. This amygdala subregion may be particularly relevant to the species difference in the effect of OT observed in this study because microstructural differences have been found between the bonobo and chimpanzee amygdala, including in this subregion (Hopkins et al., 2015; Issa et al., 2019; Rilling et al., 2012; Staes et al., 2018; Stimpson et al., 2016). Investigation of receptor structure, in particular binding affinity of OT receptors, may additionally 
reveal important species differences in light of genetic analysis showing polymorphisms in the OXTR region that differ between the two species (Staes et al., 2014).

One limitation of this study is the relatively small number of ape participants tested, mainly due to the rarity of opportunity that allowed us to do both OT administration and eye tracking with apes. There are several points to be discussed regarding this issue. First, the relatively small number of participants may have hampered the detection of significant overall species differences in the looking durations towards eyes and mouth in this study. However, we are confident that increasing the number of participants can solve this issue because 1) the previous study which included a larger number of bonobos and chimpanzees (including the ape participants tested in this study) yielded significant species difference in the looking duration to eyes, and 2) the ape participants in this study showed high intra-individual consistency in looking duration to eyes between conditions (Figure 2C) and also in comparison to the previous study (Kano et al., 2015) (Table S3). Second, the relatively small number of ape participants in this study also limits systematic examinations of individual differences. For example, among the six chimpanzees tested in this study, one chimpanzee (Iroha, a 12-years-old, mother-reared female) showed an opposite tendency from the other chimpanzees in her response to OT (Figure 2C). As we found no demographic parameter unique to this chimpanzee (Table 1), this result remains difficult to interpret. Third, sex difference is of potential interest as it is known to influence the effect of OT in human and nonhuman primates (Domes et al., 2010; Insel and Young, 2000; Jiang and Platt, 2018a, b). In this study, most ape participants were females, with one male in each species. It should be noted that these males' responses to OT were not substantially different from the females (they did not show the strongest or weakest responses in either species). Our follow-up test including stimulus sex in the model (with eye-mouth difference score as a response) did not reveal the effect of stimulus sex (or the interaction between this and other factors). However, it is worthwhile to test more directly whether 
427 OT modulates each sex's responses to the same/different sex in bonobos and chimpanzees,

428 particularly because bonobos and chimpanzees are known for distinctive dominance styles; namely,

429 chimpanzee males are generally more dominant over females, while bonobo females are often more

430 dominant than males through coordinating female coalitionary aggression towards males

431 (Tokuyama et al., 2019). It remains unclear how OT might be involved in males' and females'

432 attitudes towards different sexes in these species. Overall, the key contribution of this study is the

433 addition of the knowledge that OT affects eye contact behavior of bonobos and chimpanzees

434 differently, with some caution that such species differences could be female-biased.

Another limitation of our study is that, although we found changes in eye movement in both species after OT administration, we do not have strong external validation of the efficacy of administration procedures from hormonal measurement. Our results generally suggest that the administered OT did reach the central nervous system (and thereby caused a behavioral change), and one of our ongoing studies following the same OT administration procedures additionally found changes in eye movement patterns with a new set of stimuli in chimpanzees (Kawaguchi, personal communication). However, in our preliminary endocrinological tests examining whether OT administration increases urinary OT in chimpanzees (summarized in Supplementary material), we did not find a clear effect of OT administration, although we found some differences in the pattern of changes in urinary OT between the OT and saline conditions (sampling urine 15-90 minutes after OT administration). Our working hypothesis is that OT administered to the nasal cavity reached the central nervous system via direct nose-to-brain routes (Quintana et al., 2015) without necessarily reaching the periphery, while in previous studies with wild populations (Crockford et al., 2013;

448 Samuni et al., 2017; Wittig et al., 2014) endogenous OT trigged by relevant social behaviors was secreted into the periphery from the hypothalamus. Clearly, further studies are necessary to examine administered OT's effects on behavior and physiology in these great ape species. 
These potential limitations aside, one interesting implication from our results is that the observed opposite effect of OT on eye contact behavior may affect social interactions of bonobos and chimpanzees through the hypothesized biobehavioral feedback loop (Crockford et al., 2013; Wittig et al., 2014), and thereby promote species-typical patterns of social interaction in both species. That is, previous studies with wild bonobos and chimpanzees showed that urinary OT increased after grooming, reconciliation, food-sharing, and intergroup conflicts in chimpanzees (Crockford et al., 2013; Preis et al., 2018; Samuni et al., 2017; Wittig et al., 2014), and noncopulatory sexual contact (GG-rubbing) in bonobos (Moscovice et al., 2019). Such increases in endogenous OT may lead to different eye contact behavior in each species, as suggested in our experiments. In addition, great apes generally use eye contact to initiate and facilitate social interaction between individuals, such as an establishment of eye contact before attempting reconciliation in chimpanzees (De Waal, 1990a) and the maintenance of eye contact during GGrubbing in bonobos (Annicchiarico et al., 2020; Moscovice et al., 2019). Therefore, OT-driven changes in eye contact behavior may result in a relatively large difference in social interaction between bonobos and chimpanzees via different biobehavioral feedbacks. It remains unclear whether eye contact per se triggers OT release in bonobos and chimpanzees, as was found in dogs and humans (Nagasawa et al., 2015). Thus, further tests with bonobos and chimpanzees are required.

In conclusion, we demonstrated that OT affects eye contact behavior differently in humans' two closest living relatives, bonobos and chimpanzees. This suggests that OT may have played a modulatory role in the evolution of species-typical behavior of bonobos and chimpanzees. Furthermore, this result underscores the diversified roles of OT through phylogeny. Thus, despite the fact that OT has generally conserved roles across mammals, we should be cautious about 
Brooks et al. 25

474 generalizing results from one species to another, which echoes a message from the previous studies 475 (e.g. Insel, 2010). 
477 Acknowledgement

478 We thank the staff and apes at Kumamoto Sanctuary for their assistance in conducting the

479 experiments and Nozomi Hirayama for her help in analyzing the urinary oxytocin samples. We also

480 thank S.H. Brosnan for providing constructive comments on our manuscript. Financial support

481 came from Japan Society for Promotion of Science [KAKENHI 20H05000, 18H05072, 19 H01772

482 to FK, $18 \mathrm{H} 02489$ to MN, $19 \mathrm{H} 00972$ to TK, $19 \mathrm{H} 00629$ to SY] and Kyoto University Leading 483 Graduate Program in Primatology and Wildlife Science. 
485

486

487

488

489

490

491

492

493

494

495

496

497

498

499

500

501

502

503

504

505

506

507

508

References

Anacker, A.M., Beery, A.K., 2013. Life in groups: the roles of oxytocin in mammalian sociality.

Front Behav Neurosci 7, 185.

Andari, E., Duhamel, J.R., Zalla, T., Herbrecht, E., Leboyer, M., Sirigu, A., 2010. Promoting social behavior with oxytocin in high-functioning autism spectrum disorders. Proc Natl Acad Sci U S A $107,4389-4394$.

Anestis, S.F., Webster, T.H., Kamilar, J.M., Fontenot, M.B., Watts, D.P., Bradley, B.J., 2014. AVPR1A variation in chimpanzees (Pan troglodytes): Population differences and association with behavioral style. Int J Primatol 35, 305-324.

Annicchiarico, G., Bertini, M., Cordoni, G., Palagi, E., 2020. Look at me while having sex! Eye-toeye contact affects homosexual behaviour in bonobo females. Behaviour 1, 1-22.

Argyle, M., Dean, J., 1965. Eye-contact, distance and affiliation. Sociometry 28, 289-304.

Auyeung, B., Lombardo, M.V., Heinrichs, M., Chakrabarti, B., Sule, A., Deakin, J.B., Bethlehem,

R.A., Dickens, L., Mooney, N., Sipple, J.A., Thiemann, P., Baron-Cohen, S., 2015. Oxytocin increases eye contact during a real-time, naturalistic social interaction in males with and without autism. Transl Psychiatry 5, e507.

Barr, D.J., Levy, R., Scheepers, C., Tily, H.J., 2013. Random effects structure for confirmatory hypothesis testing: Keep it maximal. J Mem Lang 68, 255-278.

Bartz, J.A., Zaki, J., Bolger, N., Ochsner, K.N., 2011. Social effects of oxytocin in humans: context and person matter. Trends Cogn Sci 15, 301-309.

Bauman, M.D., Murai, T., Hogrefe, C.E., Platt, M.L., 2018. Opportunities and challenges for intranasal oxytocin treatment studies in nonhuman primates. Am J Primatol 80, e22913.

Burkett, J.P., Andari, E., Johnson, Z.V., Curry, D.C., de Waal, F.B., Young, L.J., 2016. Oxytocindependent consolation behavior in rodents. Science 351, 375-378. 
509 Crockford, C., Wittig, R.M., Langergraber, K., Ziegler, T.E., Zuberbuhler, K., Deschner, T., 2013.

510 Urinary oxytocin and social bonding in related and unrelated wild chimpanzees. Proc Biol Sci 280,

51120122765.

512 Dal Monte, O., Noble, P.L., Costa, V.D., Averbeck, B.B., 2014. Oxytocin enhances attention to the 513 eye region in rhesus monkeys. Front Neurosci 8, 41.

514 De Dreu, C.K., Greer, L.L., Handgraaf, M.J., Shalvi, S., Van Kleef, G.A., Baas, M., Ten Velden, 515 F.S., Van Dijk, E., Feith, S.W., 2010. The neuropeptide oxytocin regulates parochial altruism in 516 intergroup conflict among humans. Science 328, 1408-1411.

517 De Waal, F.B.M., 1990a. Peacemaking among primates. Harvard University Press, Cambridge.

518 De Waal, F.B.M., 1990b. Sociosexual behavior used for tension regulation in all age and sex

519 combinations among bonobos, Pedophilia. Springer, New York, pp. 378-393.

520 Domes, G., Lischke, A., Berger, C., Grossmann, A., Hauenstein, K., Heinrichs, M., Herpertz, S.C.,

521 2010. Effects of intranasal oxytocin on emotional face processing in women.

522 Psychoneuroendocrinology 35, 83-93.

523 Ebitz, R.B., Watson, K.K., Platt, M.L., 2013. Oxytocin blunts social vigilance in the rhesus

524 macaque. Proc Natl Acad Sci U S A 110, 11630-11635.

525 Emery, N.J., 2000. The eyes have it: the neuroethology, function and evolution of social gaze.

526 Neurosci Biobehav Rev 24, 581-604.

527 Engqvist, L., 2005. The mistreatment of covariate interaction terms in linear model analyses of 528 behavioural and evolutionary ecology studies. Anim Behav 70.

529 Ferrari, P.F., Paukner, A., Ionica, C., Suomi, S.J., 2009. Reciprocal face-to-face communication

530 between rhesus macaque mothers and their newborn infants. Curr Biol 19, 1768-1772.

531 Gamer, M., Zurowski, B., Buchel, C., 2010. Different amygdala subregions mediate valence-related and attentional effects of oxytocin in humans. Proc Natl Acad Sci U S A 107, 9400-9405. 
533 Guastella, A.J., Mitchell, P.B., Dadds, M.R., 2008. Oxytocin increases gaze to the eye region of 534 human faces. Biol Psychiatry 63, 3-5.

535 Hall, K., Smith, M., Russell, J.L., Lambeth, S.P., Schapiro, S.J., Brosnan, S.F., 2019. Chimpanzees

536 rarely settle on consistent patterns of play in the hawk dove, assurance, and prisoner's dilemma

537 games, in a token exchange task. Anim Behav Cogn 6, 48-70.

538 Hammock, E.A., Young, L.J., 2005. Microsatellite instability generates diversity in brain and 539 sociobehavioral traits. Science 308, 1630-1634.

540 Harari-Dahan, O., Bernstein, A., 2014. A general approach-avoidance hypothesis of oxytocin:

541 accounting for social and non-social effects of oxytocin. Neurosci Biobehav Rev 47, 506-519.

542 Harari-Dahan, O., Bernstein, A., 2017. Oxytocin attenuates social and non-social avoidance: Re-

543 thinking the social specificity of Oxytocin. Psychoneuroendocrinology 81, 105-112.

544 Hare, B., Wobber, V., Wrangham, R., 2012. The self-domestication hypothesis: evolution of

545 bonobo psychology is due to selection against aggression. Anim Behav 83, 573-585.

546 Hare, B., Yamamoto, S., 2017. Minding the bonobo mind, in: Hare, B., Yamamoto, S. (Eds.),

547 Bonobos: Unique in Mind, Brain and Behavior. Oxford University Press, Oxford, pp. 1-13.

548 Herbeck, Y.E., Gulevich, R.G., 2019. Neuropeptides as facilitators of domestication. Cell Tissue

$549 \quad$ Res 375, 295-307.

550 Herrmann, E., Hare, B., Call, J., Tomasello, M., 2010. Differences in the cognitive skills of bonobos 551 and chimpanzees. PLOS ONE 5, e12438.

552 Hopkins, W.D., Donaldson, Z.R., Young, L.J., 2012. A polymorphic indel containing the RS3

553 microsatellite in the $5^{\prime}$ flanking region of the vasopressin V1a receptor gene is associated with

554 chimpanzee (Pan troglodytes) personality. Genes, Brain and Behav 11, 552-558.

555 Hopkins, W.D., Schaeffer, J., Russell, J.L., Bogart, S.L., Meguerditchian, A., Coulon, O., 2015. A

556 comparative assessment of handedness and its potential neuroanatomical correlates in chimpanzees 
557

558

(Pan troglodytes) and bonobos (Pan paniscus), in: Brian, H., Shinya, Y. (Eds.), Bonobo Cognition and Behaviour. Brill, Leiden, pp. 215-246.

Insel, T.R., 2010. The challenge of translation in social neuroscience: a review of oxytocin, vasopressin, and affiliative behavior. Neuron $65,768-779$.

Insel, T.R., Shapiro, L.E., 1992. Oxytocin receptor distribution reflects social organization in monogamous and polygamous voles. Proc Natl Acad Sci U S A 89, 5981-5985.

Insel, T.R., Young, L.J., 2000. Neuropeptides and the evolution of social behavior. Curr Opin Neurobiol 10, 784-789.

Israel, S., Lerer, E., Shalev, I., Uzefovsky, F., Riebold, M., Laiba, E., Bachner-Melman, R., Maril, A., Bornstein, G., Knafo, A., 2009. The oxytocin receptor (OXTR) contributes to prosocial fund allocations in the dictator game and the social value orientations task. PloS One 4, e5535. Issa, H.A., Staes, N., Diggs-Galligan, S., Stimpson, C.D., Gendron-Fitzpatrick, A., Taglialatela, J.P., Hof, P.R., Hopkins, W.D., Sherwood, C.C., 2019. Comparison of bonobo and chimpanzee brain microstructure reveals differences in socio-emotional circuits. Brain Struct Funct 224, 239251.

Jiang, Y., Platt, M.L., 2018a. Oxytocin and vasopressin flatten dominance hierarchy and enhance behavioral synchrony in part via anterior cingulate cortex. Sci Rep 8, 8201.

Jiang, Y., Platt, M.L., 2018b. Oxytocin and vasopressin increase male-directed threats and vocalizations in female macaques. Sci Rep 8, 18011.

Kano, F., Hirata, S., Call, J., 2015. Social attention in the two species of pan: Bonobos make more eye contact than chimpanzees. PLoS One 10, e0129684.

Kano, F., Hirata, S., Call, J., Tomonaga, M., 2011. The visual strategy specific to humans among hominids: a study using the gap-overlap paradigm. Vision Res 51, 2348-2355. 
Kemp, A.H., Guastella, A.J., 2011. The role of oxytocin in human affect: a novel hypothesis. Curr Dir Psychol Sci 20, 222-231.

Kosfeld, M., Heinrichs, M., Zak, P.J., Fischbacher, U., Fehr, E., 2005. Oxytocin increases trust in humans. Nature 435, 673-676.

Kotani, M., Shimono, K., Yoneyama, T., Nakako, T., Matsumoto, K., Ogi, Y., Konoike, N., Nakamura, K., Ikeda, K., 2017. An eye tracking system for monitoring face scanning patterns reveals the enhancing effect of oxytocin on eye contact in common marmosets.

Psychoneuroendocrinology 83, 42-48.

Krupenye, C., Kano, F., Hirata, S., Call, J., Tomasello, M., 2016. Great apes anticipate that other individuals will act according to false beliefs. Science 354, 110-114.

MacDonald, E., Dadds, M.R., Brennan, J.L., Williams, K., Levy, F., Cauchi, A.J., 2011. A review of safety, side-effects and subjective reactions to intranasal oxytocin in human research.

Psychoneuroendocrinology 36, 1114-1126.

Macdonald, K., Macdonald, T.M., 2010. The peptide that binds: a systematic review of oxytocin and its prosocial effects in humans. Harv Rev Psychiatry 18, 1-21.

Modi, M.E., Connor-Stroud, F., Landgraf, R., Young, L.J., Parr, L.A., 2014. Aerosolized oxytocin increases cerebrospinal fluid oxytocin in rhesus macaques. Psychoneuroendocrinology 45, 49-57.

Moscovice, L.R., Surbeck, M., Fruth, B., Hohmann, G., Jaeggi, A.V., Deschner, T., 2019. The cooperative sex: Sexual interactions among female bonobos are linked to increases in oxytocin, proximity and coalitions. Horm Behav 116, 104581.

Mulholland, M.M., Mahovetz, L.M., Mareno, M.C., Reamer, L.A., Schapiro, S.J., Hopkins, W.D., 2020. Differences in the mutual eye gaze of bonobos (Pan paniscus) and chimpanzees (Pan troglodytes). J Comp Psychol 134, 318-322. 
603

604

605

606

607

608

609

610

611

612

613

614

615

616

617

618

619

620

621

622

623

624

625

Nagasawa, M., Mitsui, S., En, S., Ohtani, N., Ohta, M., Sakuma, Y., Onaka, T., Mogi, K., Kikusui, T., 2015. Oxytocin-gaze positive loop and the coevolution of human-dog bonds. Science 348, 333336.

Parr, L.A., Modi, M., Siebert, E., Young, L.J., 2013. Intranasal oxytocin selectively attenuates rhesus monkeys' attention to negative facial expressions. Psychoneuroendocrinology 38, 1748-1756.

Preis, A., Samuni, L., Mielke, A., Deschner, T., Crockford, C., Wittig, R.M., 2018. Urinary oxytocin levels in relation to post-conflict affiliations in wild male chimpanzees (Pan troglodytes verus). Horm Behav 105, 28-40.

Proctor, D., Calcutt, S.E., Burke, K., de Waal, F.B.M., 2016. Intranasal oxytocin failed to affect chimpanzee (Pan troglodytes) social behavior. Anim Behav Cogn 3, 150-158.

Prufer, K., Munch, K., Hellmann, I., Akagi, K., Miller, J.R., Walenz, B., Koren, S., Sutton, G., Kodira, C., Winer, R., Knight, J.R., Mullikin, J.C., Meader, S.J., Ponting, C.P., Lunter, G., Higashino, S., Hobolth, A., Dutheil, J., Karakoc, E., Alkan, C., Sajjadian, S., Catacchio, C.R., Ventura, M., Marques-Bonet, T., Eichler, E.E., Andre, C., Atencia, R., Mugisha, L., Junhold, J., Patterson, N., Siebauer, M., Good, J.M., Fischer, A., Ptak, S.E., Lachmann, M., Symer, D.E., Mailund, T., Schierup, M.H., Andres, A.M., Kelso, J., Paabo, S., 2012. The bonobo genome compared with the chimpanzee and human genomes. Nature 486, 527-531.

Putnam, P.T., Roman, J.M., Zimmerman, P.E., Gothard, K.M., 2016. Oxytocin enhances gazefollowing responses to videos of natural social behavior in adult male rhesus monkeys.

Psychoneuroendocrinology 72, 47-53.

Quintana, D.S., Alvares, G.A., Hickie, I.B., Guastella, A.J., 2015. Do delivery routes of intranasally administered oxytocin account for observed effects on social cognition and behavior? A two-level model. Neurosci. Biobehav. Rev. 49, 182-192. 
626

627

628

629

630

631

632

633

634

635

636

637

638

639

640

641

642

643

644

645

646

647

Rilling, J.K., Scholz, J., Preuss, T.M., Glasser, M.F., Errangi, B.K., Behrens, T.E., 2012.

Differences between chimpanzees and bonobos in neural systems supporting social cognition. Soc

Cogn Affect Neurosci 7, 369-379.

Rodrigues, S.M., Saslow, L.R., Garcia, N., John, O.P., Keltner, D., 2009. Oxytocin receptor genetic variation relates to empathy and stress reactivity in humans. Proc Natl Acad Sci U S A 106, $21437-$ 21441.

Rogers-Carter, M.M., Varela, J.A., Gribbons, K.B., Pierce, A.F., McGoey, M.T., Ritchey, M., Christianson, J.P., 2018. Insular cortex mediates approach and avoidance responses to social affective stimuli. Nat Neurosci 21, 404-414.

Romero, T., Nagasawa, M., Mogi, K., Hasegawa, T., Kikusui, T., 2014. Oxytocin promotes social bonding in dogs. Proc Natl Acad Sci U S A 111, 9085-9090.

Samuni, L., Preis, A., Deschner, T., Crockford, C., Wittig, R.M., 2018. Reward of labor coordination and hunting success in wild chimpanzees. Commun Biol 1, 138.

Samuni, L., Preis, A., Mundry, R., Deschner, T., Crockford, C., Wittig, R.M., 2017. Oxytocin reactivity during intergroup conflict in wild chimpanzees. Proc Natl Acad Sci U S A 114, 268-273.

Schielzeth, H., 2010. Simple means to improve the interpretability of regression coefficients.

Methods Ecol Evol 1, 103-113.

Shamay-Tsoory, S.G., Abu-Akel, A., 2016. The social salience hypothesis of oxytocin. Biol Psychiatry 79, 194-202.

Shamay-Tsoory, S.G., Fischer, M., Dvash, J., Harari, H., Perach-Bloom, N., Levkovitz, Y., 2009. Intranasal administration of oxytocin increases envy and schadenfreude (gloating). Biol Psychiatry 66, 864-870. 
648

649

650

651

652

653

654

655

656

657

658

659

660

661

662

663

664

665

666

667

668

669

Simpson, E.A., Sclafani, V., Paukner, A., Hamel, A.F., Novak, M.A., Meyer, J.S., Suomi, S.J., Ferrari, P.F., 2014. Inhaled oxytocin increases positive social behaviors in newborn macaques. Proc Natl Acad Sci U S A 111, 6922-6927.

Staes, N., Smaers, J.B., Kunkle, A.E., Hopkins, W.D., Bradley, B.J., Sherwood, C.C., 2018.

Evolutionary divergence of neuroanatomical organization and related genes in chimpanzees and bonobos. Cortex 118, 154-164.

Staes, N., Stevens, J.M., Helsen, P., Hillyer, M., Korody, M., Eens, M., 2014. Oxytocin and vasopressin receptor gene variation as a proximate base for inter- and intraspecific behavioral differences in bonobos and chimpanzees. PLoS One 9, e113364.

Staes, N., Weiss, A., Helsen, P., Korody, M., Eens, M., Stevens, J.M.G., 2016. Bonobo personality traits are heritable and associated with vasopressin receptor gene 1a variation. Sci Rep 6, 38193. Stimpson, C.D., Barger, N., Taglialatela, J.P., Gendron-Fitzpatrick, A., Hof, P.R., Hopkins, W.D., Sherwood, C.C., 2016. Differential serotonergic innervation of the amygdala in bonobos and chimpanzees. Soc Cogn Affect Neurosci 11, 413-422.

Tan, J., Hare, B., 2013. Bonobos share with strangers. PLoS One 8, e51922.

Thomsen, C.E., 1974. Eye contact by non-human primates toward a human observer. Anim Behav $22,144-149$.

Tokuyama, N., Sakamaki, T., Furuichi, T., 2019. Inter-group aggressive interaction patterns indicate male mate defense and female cooperation across bonobo groups at Wamba, Democratic Republic of the Congo. Am J Phys Anthropol 170, 535-550.

Warton, D.I., Hui, F.K., 2011. The arcsine is asinine: the analysis of proportions in ecology.

Ecology 92, 3-10. 
670 Wilson, V.A., Weiss, A., Humle, T., Morimura, N., Udono, T., Idani, G., Matsuzawa, T., Hirata, S.,

671 Inoue-Murayama, M., 2017. Chimpanzee personality and the arginine vasopressin receptor 1A

672 genotype. Behav Genet 47, 215-226.

673 Wittig, R.M., Crockford, C., Deschner, T., Langergraber, K.E., Ziegler, T.E., Zuberbuhler, K.,

674 2014. Food sharing is linked to urinary oxytocin levels and bonding in related and unrelated wild 675 chimpanzees. Proc Biol Sci 281, 20133096.

676 
Table S1. Presentation duration of each AOI in each video (sec). For Body (and Screen) AOI, the presentation duration was maximal ( $3 \mathrm{~min})$.

\begin{tabular}{lrlrr}
\hline Video & Eye/Mouth & Face & Action target & Genital \\
Bonobo video 1 & 105.1 & 165.5 & 41.8 & 48.6 \\
Bonobo video 2 & 109.1 & 163.6 & 54.2 & 48.0 \\
Chimp video 1 & 105.2 & 163.8 & 33.3 & 39.6 \\
Chimp video 2 & 114.1 & 167.3 & 11.8 & 59.9 \\
\hline
\end{tabular}

Table S2. The model results for looking durations (sec) to each Areas-Of-Interest. The main effects of each term were examined when the interaction term was not significant (but were kept when they were significant or marginally significant). Intercepts were from the full models (with the interaction terms). Confidence intervals (CI) were from the parametric bootstrapping method with 1000 replicates. See Figure 2 and S2 for the means (and CIs) of looking duration to each Areas-Of-Interest. ${ }^{*} \mathrm{p}<0.05,+\mathrm{p}$ $<0.1$.

\begin{tabular}{llcccccc}
\hline Response & \multicolumn{1}{c}{ Term } & Estimate & $\mathrm{SE}$ & $\mathrm{CI}_{\text {lower }}$ & $\mathrm{Cl}_{\text {upper }}$ & $\chi^{2}$ & $\mathrm{p}$ \\
Eye & Intercept & 17.8 & 3.6 & 10.4 & 25.2 & - & - \\
& Species:Condition & -3.4 & 1.4 & -6.2 & -0.4 & 4.9 & $0.028\left(^{*}\right)$ \\
Mouth & Intercept & 15.9 & 2.8 & 10.4 & 21.6 & - & - \\
& Species:Condition & 3.6 & 1.9 & -0.09 & 7.5 & 3.2 & $0.072\left(^{+}\right)$ \\
Eye minus & Intercept & 2.0 & 5.3 & -8.6 & 12.6 & - & - \\
mouth & Species:Condition & -6.9 & 2.4 & -12.0 & -2.6 & 6.4 & $0.011\left(^{*}\right)$ \\
& & & & & & & \\
Face (as a & Intercept & 53.4 & 5.5 & 42.3 & 64.3 & - & - \\
whole) & Species:Condition & 1.3 & 3.9 & -6.6 & 9.6 & 0.1 & 0.74 \\
& Species & -2.3 & 7.3 & -17.4 & 12.9 & 0.1 & 0.75 \\
& Condition & -0.7 & 1.9 & -4.8 & 3.0 & 0.1 & 0.73 \\
Action target & Intercept & -2.4 & 0.3 & -3.0 & -1.9 & - & - \\
\#2 & Species:Condition & 0.4 & 0.3 & -0.2 & 0.9 & 2.2 & 0.14 \\
& $\quad$ Species & 1.0 & 0.4 & 0.2 & 1.8 & 4.4 & $0.036\left(^{*}\right)$ \\
& Condition & -0.2 & 0.1 & -0.5 & 0.1 & 2.1 & 0.15 \\
& Intercept & -2.3 & 0.2 & -2.8 & -1.9 & - & - \\
Genital ${ }^{* 2}$ & Species:Condition & 0.1 & 0.2 & -0.3 & 0.7 & 0.3 & 0.56 \\
& & & & & & &
\end{tabular}




\begin{tabular}{llcccccc} 
& Species & -0.4 & 0.2 & -0.9 & 0.09 & 2.4 & 0.12 \\
& Condition & -0.1 & 0.1 & -0.3 & 0.1 & 0.7 & 0.40 \\
$\begin{array}{l}\text { Body (as a } \\
\text { whole) }\end{array}$ & Intercept & 56.4 & 5.0 & 45.7 & 67.4 & - & - \\
& Species:Condition & 1.6 & 3.4 & -5.6 & 8.5 & 0.3 & 0.61 \\
& Species & 0.9 & 6.8 & -11.5 & 12.8 & 0.02 & 0.89 \\
& Condition & -0.8 & 1.7 & -3.9 & 2.2 & 0.2 & 0.65 \\
Screen (as a & Intercept & 106.7 & 9.4 & 87.3 & 125.7 & - & - \\
whole) & Species:Condition & 1.6 & 5.7 & -9.3 & 13.3 & 0.08 & 0.77 \\
& Species & 31.7 & 12.8 & 4.8 & 58.6 & 4.9 & $0.028\left(^{*}\right)$ \\
& Condition & -2.5 & 2.8 & -8.0 & 3.4 & 0.8 & 0.38 \\
\hline
\end{tabular}

\#1 All degrees of freedom were 1.

\#2 For the action-target and genital AOIs, the looking duration was divided by the total presentation duration, and then logit-transformed.

Table S3. The comparison of participants' difference scores (indicated as 'Diff.') as the total looking duration $(\mathrm{sec})$ for mouth subtracted from that for eyes in all trials (6 minutes in this study, and 4.5 minutes in the previous study) across conditions in this study and between this and previous study (Kano, Hirata, \& Call, 2015). Also shown is the rank according to the difference scores to guide this comparison. See the details about participants in Table 1.

\begin{tabular}{lccccccc}
\hline Species & Name & \multicolumn{2}{c}{ Oxytocin condition } & \multicolumn{2}{c}{ Saline condition } & \multicolumn{2}{c}{ Previous study } \\
& & Diff. & Rank & Diff. & Rank & Diff. & Rank \\
Bonobo & Ikela & 38.76 & 2 & 30.91 & 2 & 99.45 & 1 \\
Bonobo & Connie-Lenore & -31.33 & 10 & -33.57 & 11 & 0.27 & 9 \\
Bonobo & Louise & 18.82 & 3 & 7.99 & 4 & 62.55 & 3 \\
Bonobo & Lolita & -22.28 & 9 & -31.05 & 10 & -16.2 & 11 \\
Bonobo & Vijay & 48.67 & 1 & 46.31 & 1 & 95.94 & 2 \\
Chimpanzee & Hatsuka & -7.27 & 7 & 2.96 & 6 & -7.2 & 10 \\
Chimpanzee & Iroha & -1.37 & 6 & -9.22 & 8 & 23.94 & 6 \\
Chimpanzee & Zamba & -53.52 & 11 & -30.01 & 9 & 8.73 & 8 \\
Chimpanzee & Misaki & 14.29 & 4 & 16.76 & 3 & 27.81 & 5 \\
Chimpanzee & Mizuki & -14.19 & 8 & -3.13 & 7 & 35.46 & 4
\end{tabular}




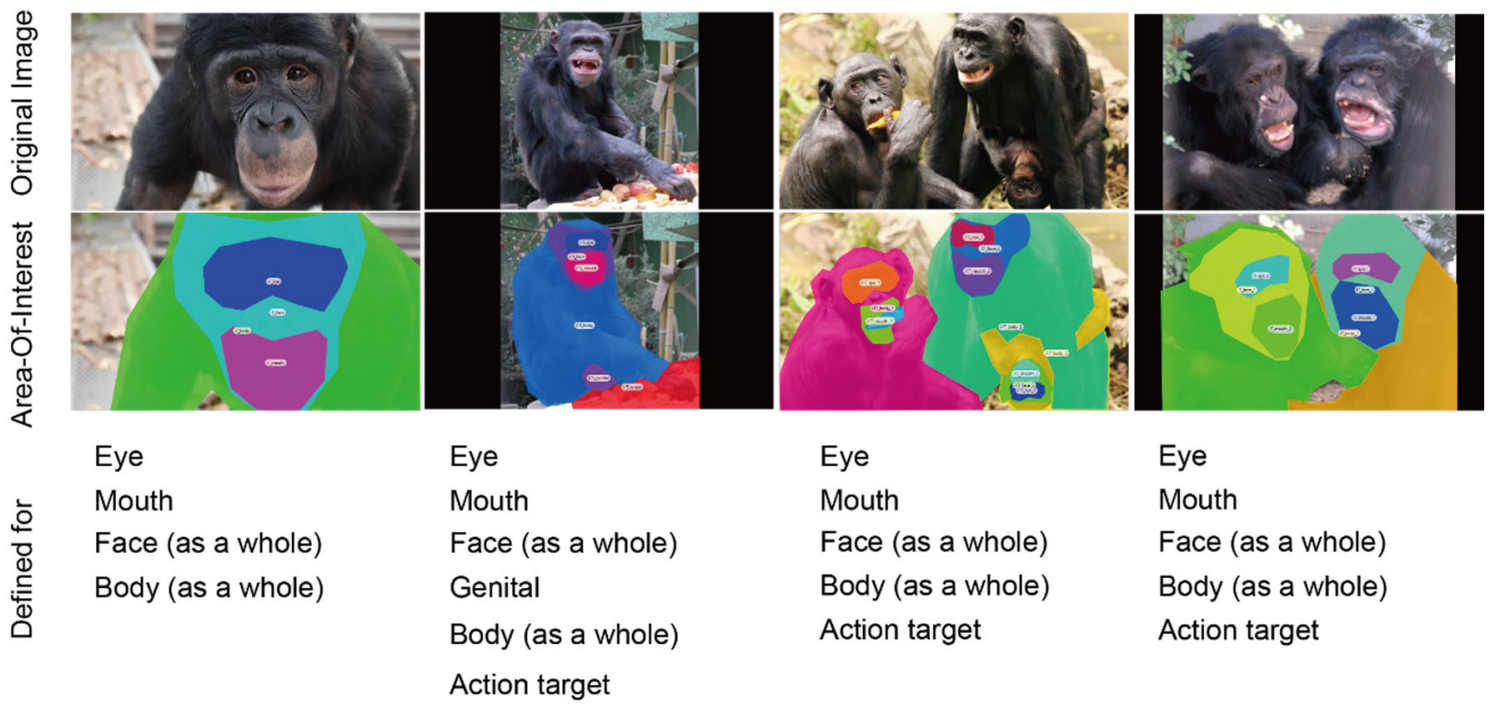

Figure S1. Examples for Areas-Of-Interests (AOIs) defined for the images. 

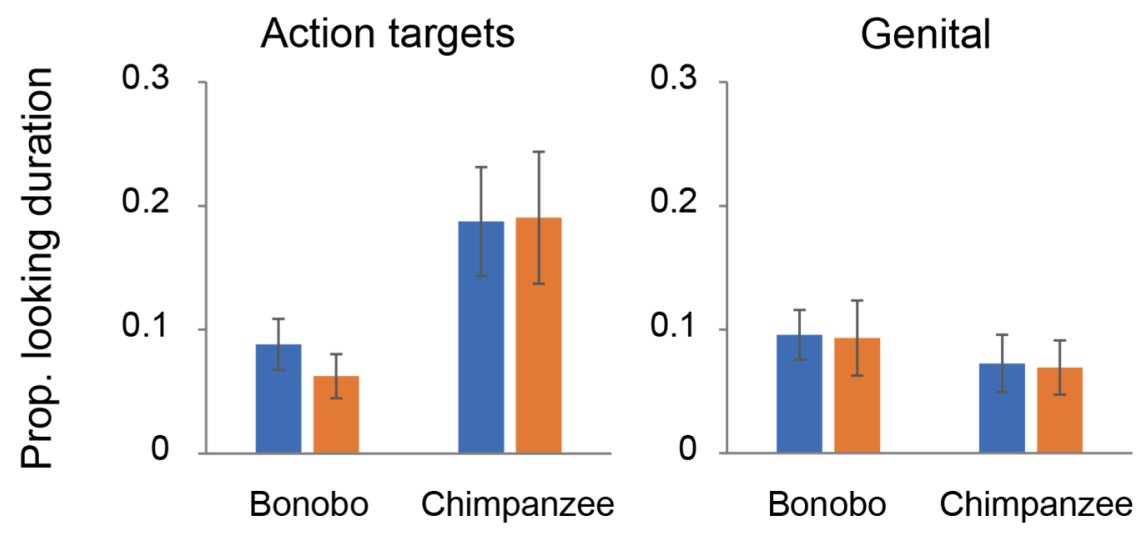

Face (as a whole)

Body (as a whole)
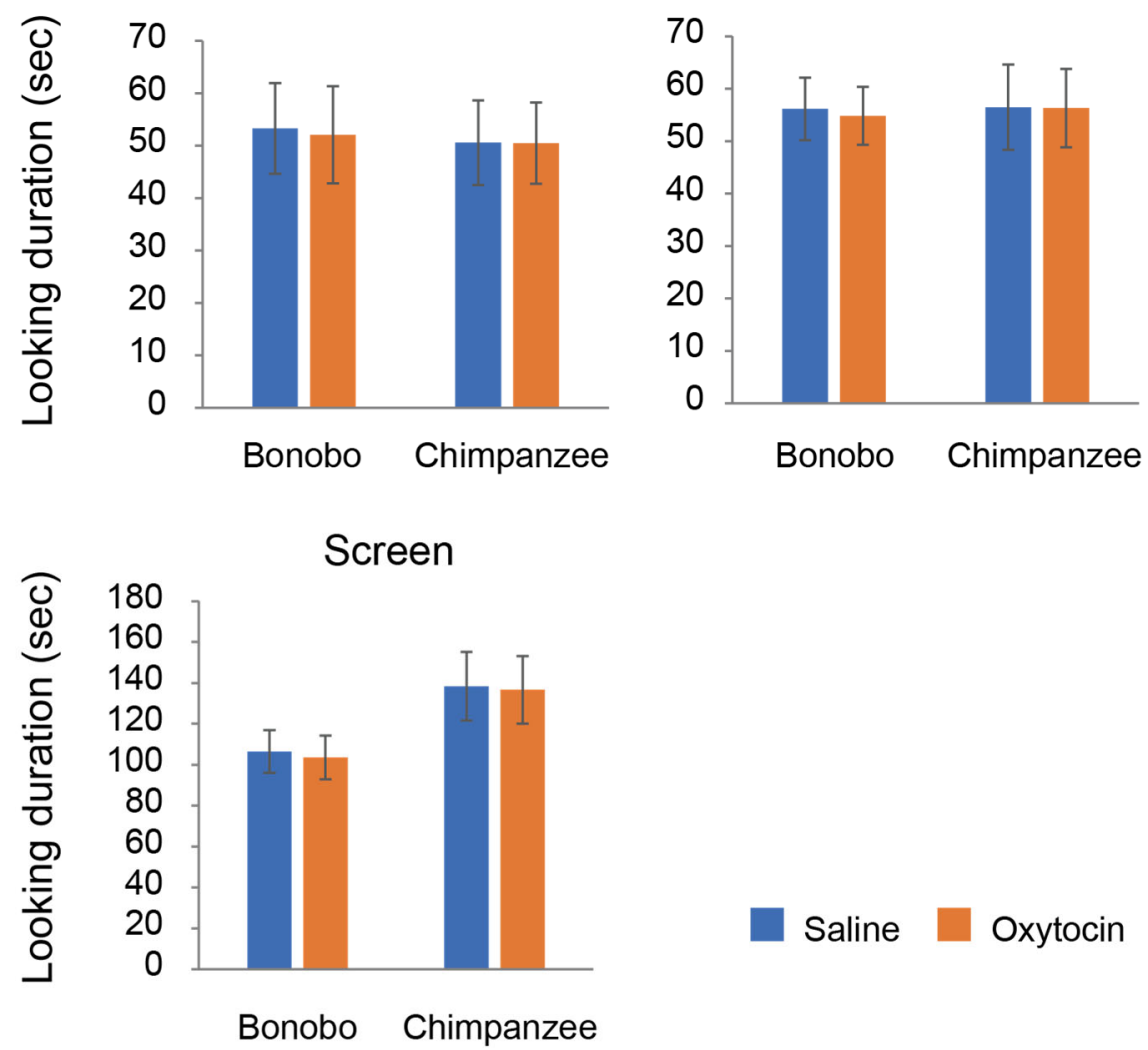

Saline Oxytocin

Figure S2. Mean looking durations (sec) for the action targets, face (as a whole), genital, and body (as a whole) in each trial (presenting a 3-min video). Error bars denote $95 \%$ confidence intervals. For the action-target and genital AOIs, the looking duration to each AOI was divided by the total presentation duration of AOI (and then logit-transformed in LMM). 


\section{Effect of stimulus properties.}

We tested the effect of stimulus properties (facial expressions, gaze directions, group affiliation, and stimulus sex) in the model by restructuring the dataset and adding each stimulus property term to the original models: Eye-mouth difference score $\sim$ Species*Condition*Stimulus property + Block/Trial + $(1+$ Condition*Stimulus property + Block/Trial $\|$ Subject $)$. The model checks were performed as described in the main text. We dropped the interaction terms if they were not significant and then tested the lower-order interaction terms or the main effects of respective terms.

Ingroup vs. outgroup face: We included the group affiliation (ingroup, outgroup) into the model as a stimulus property term. The three-way interaction effect, and the two-way interaction effects between group affiliation and condition (this was marginally at $\mathrm{p}<0.1 ; \beta=2.4, \mathrm{SE}=1.4, \mathrm{CI}_{\text {lower }}=-0.4, \mathrm{CI}_{\text {upper }}$ $=5.2, \chi^{2}=2.7, p=0.098$ ), and between group affiliation and species were not significant (and thus these were dropped from the model). We found a significant interaction effect between species and condition $\left(\beta=-2.8, \mathrm{SE}=1.1, \mathrm{CI}_{\text {lower }}=-5.3, \mathrm{CI}_{\text {upper }}=-0.37, \chi^{2}=4.7, \mathrm{p}=0.030\right)$, confirming the results described in the main text. Additionally, we found a significant effect of group affiliation ( $\beta=-4.1$, SE $\left.=1.1, \mathrm{CI}_{\text {lower }}=-6.4, \mathrm{CI}_{\text {upper }}=-1.8, \chi^{2}=7.8, \mathrm{p}=0.0053\right)$. The latter result and Figure $\mathrm{S} 3$ indicated that both species viewed the eyes of ingroup faces longer than those of outgroup faces.

Male vs. female face: We included the stimulus sex (male, female) into the model as the stimulus property term. The three-way interaction effect, and the two-way interaction effects between stimulus sex and condition, and between stimulus sex and species were not significant (and thus dropped from the model). We found a significant interaction effect between species and condition $(\beta=-2.8, \mathrm{SE}=$ $\left.1.2, \mathrm{CI}_{\text {lower }}=-5.1, \mathrm{CI}_{\text {upper }}=-0.5, \chi^{2}=4.6, \mathrm{p}=0.032\right)$, confirming the results described in the main text. The main effect of stimulus sex was not significant $\left(\beta=-0.7, \mathrm{SE}=0.9, \mathrm{CI}_{\text {lower }}=-2.6, \mathrm{CI}_{\text {upper }}=1.1, \chi^{2}\right.$ $=0.2, p=0.69$ ). Although we could not test the effect of participant sex due to small number of males, excluding the two males, one male from each species (Vijay and Zamba), from the model yielded similar results. 
Direct vs. averted gaze face: We included the gaze directions of faces (direct, averted) into the model as a stimulus property term. The three-way interaction effect was significant $\left(\beta=-6.7, \mathrm{SE}=2.5, \mathrm{CI}_{\text {lower }}\right.$ $\left.=-11.5, \mathrm{CI}_{\text {upper }}=-1.9, \chi^{2}=5.7, \mathrm{p}=0.017\right)$. The separate analyses by gaze direction revealed that $\mathrm{OT}$ affected bonobos and chimpanzees differentially when they viewed the faces with direct gaze $(\beta=-$ $\left.6.8, \mathrm{SE}=2.0, \mathrm{CI}_{\text {lower }}=-10.6, \mathrm{CI}_{\text {upper }}=-3.0, \chi^{2}=8.3, \mathrm{p}=0.0040\right)$, but not when they viewed the faces with averted gaze $\left(\beta=-0.14, \mathrm{SE}=1.1, \mathrm{CI}_{\text {lower }}=-2.5, \mathrm{CI}_{\text {upper }}=2.2, \chi^{2}=0.02, \mathrm{p}=0.90\right)$. These results consolidated the idea that OT differentially modulated the two species' eye contact behavior (i.e. looks to direct gaze).

Facial expressions, neutral face, play face vs. grimace: We included the expressions of faces (neutral face, play face, grimace) into the model as a stimulus property term. The three-way interaction effect, and the two-way interaction effects between stimulus facial expression and condition, and between facial expression and species were not significant (and thus dropped from the model). We found a significant interaction effect between species and condition $\left(\beta=-1.9, \mathrm{SE}=0.7, \mathrm{CI}_{\text {lower }}=-3.4, \mathrm{CI}_{\text {upper }}\right.$ $=-0.5, \chi^{2}=5.9, p=0.015$ ), confirming the results described in the main text. The main effect of facial expression was also significant (neutral vs. grimace, $\beta=-6.8, \mathrm{SE}=1.9, \mathrm{CI}_{\text {lower }}=-10.6, \mathrm{CI}_{\text {upper }}=-3.5$; ; $\chi^{2}=8.4, \mathrm{p}=0.004 ;$ neutral vs. play face, $\beta=-6.2, \mathrm{SE}=1.9, \mathrm{CI}_{\text {lower }}=-9.7, \mathrm{CI}_{\text {upper }}=-2.2 ; \chi^{2}=7.1, \mathrm{p}=$ 0.008). These results and Figure S3 additionally indicated that both species viewed the mouths of emotional facial expressions longer than those of neutral faces. 
A
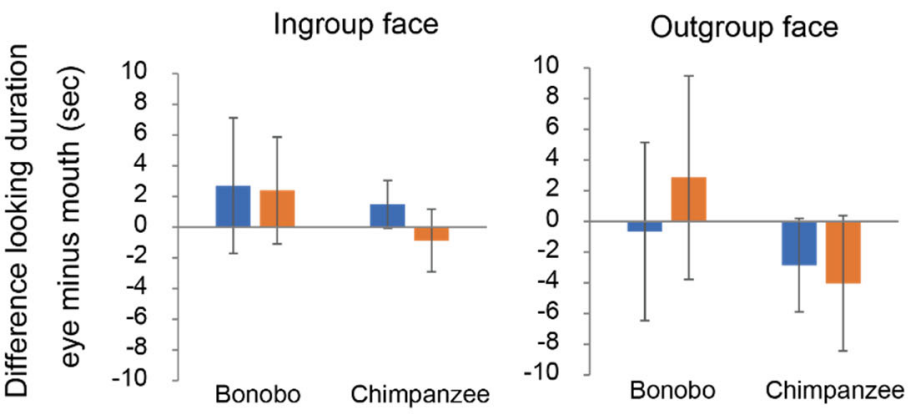

Saline Oxytocin

B
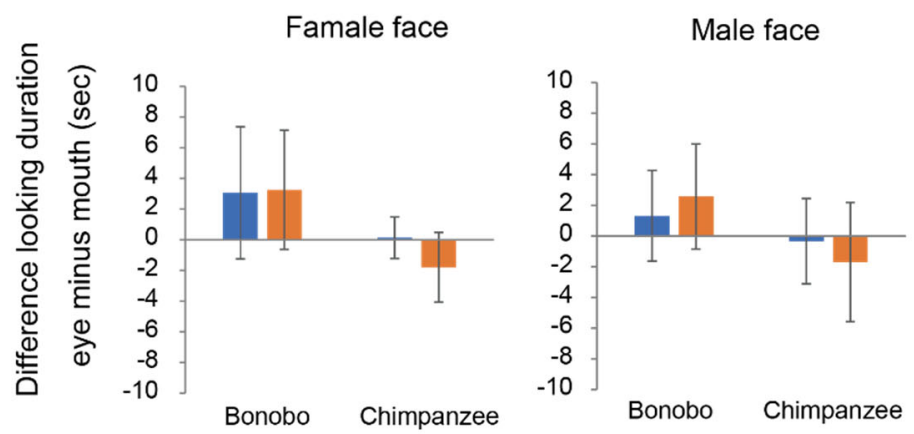

Saline Oxytocin

C
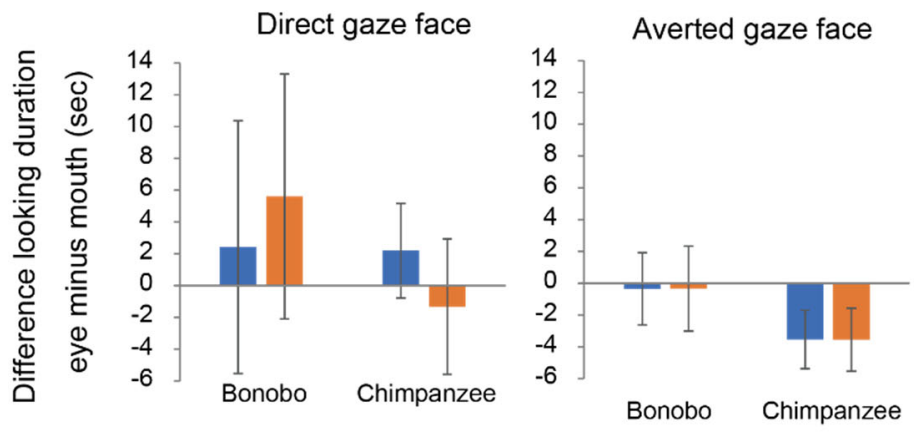

D
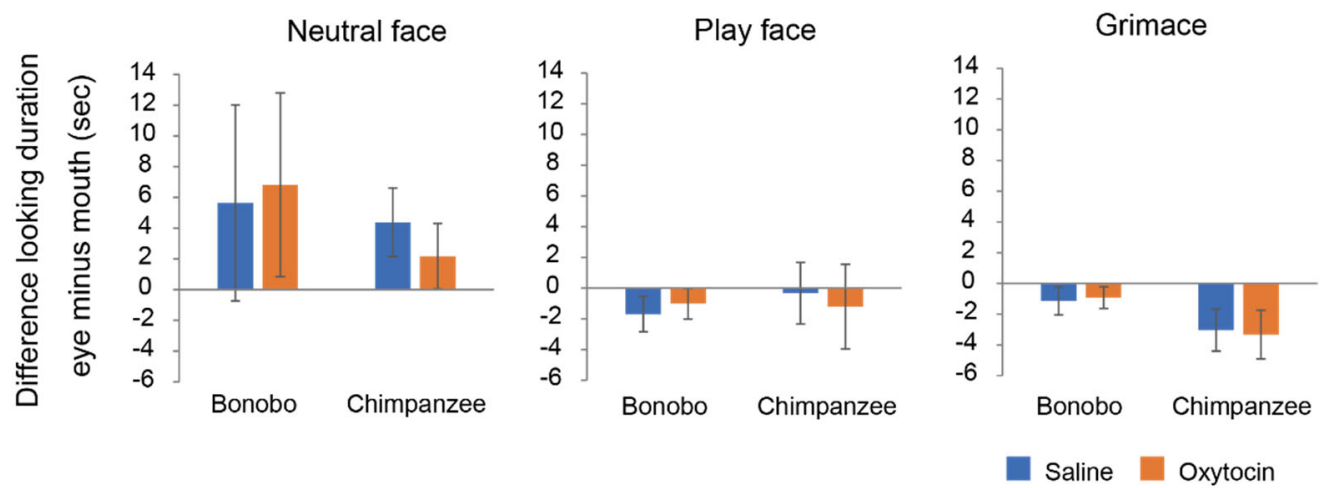

Figure S3. Mean eye-mouth difference looking duration (in sec, per trial) by each stimulus property; group affiliation (A), stimulus sex (B), gaze direction (C) and facial expression (D). Error bars denote $95 \%$ confidence intervals. 


\section{Checking the results with different model structures}

One reviewer questioned whether we should use viewing time per stimulus (picture/scene, $\sim 3 \mathrm{~s}$ ), not the sum of viewing time per each movie (180s), as one data point and then include stimulus ID as an additional random effect in the model, in order to secure a larger dataset and information pertaining to different stimuli. We decided to use the latter unit mainly because there were too many zeros in the data when formatted in the former unit, especially in the viewing times for the eye and mouth AOIs; this violates the assumption of homogeneity of variance in LMM and led us to make more complex models (more specifically, such responses essentially include two kinds of response, looked/nonlooked, if looked, how long it was). Additionally, previous eye-tracking studies on which we modelled our study have used the latter unit (Kano et al., 2015; Kano et al., 2018). However, it may be important to check if our results are not confined to any particular model structure. Fortunately, we could use the former unit with one of our key models testing the species difference in the effect of OT on eye contact, namely, the model which used eye-minus-mouth viewing times by excluding cases in which the participant viewed neither eye nor mouth in a given stimulus (excluding 0 minus 0 to be distinguished from $\mathrm{X}$ minus $\mathrm{X}$; this could be done because the main purpose of this analysis is to examine the shift of attention from eye/mouth to mouth/eye in the OT compared to the saline condition). In this analysis, we used the model formula: Response (eye-minus-mouth) $\sim$ Species * Condition + Block/Trial $+(1+$ Condition + Block/Trial $\mid$ Subject $)+(1+$ Condition + Trial |Stimulus ID $))$. The model checks were performed in the same way as described in the main text. We similarly found a significant interaction between species and condition $\left(\beta=-0.18, \mathrm{SE}=0.08, \mathrm{CI}_{\text {lower }}=-0.39, \mathrm{CI}_{\text {upper }}=-0.002, \chi^{2}=5.3, \mathrm{p}=\right.$ 0.022), confirming the results described in the main text.

\section{Urinary analysis}

Summary: We examined if urinary OT levels differentially increase after administration of OT and saline placebo. We particularly took the sampling timings after administration of OT/Saline with caution and conducted three tests (Test 1-3) which varied the sampling timings (Test 1: 90-120 min, 
Test 2: 15-90 min, Test 3: 15-60 min, since the end time of administration). Overall, we did not find a clear OT effect in all three tests, although we found a marginally significant effect suggesting a differential pattern of change over time in urinary OT between conditions when we combined the data from the latter two tests (Test 2-3; i.e. 15-90 $\mathrm{min}$ ). This unclarity in urinary analysis raised one concern that OT was not adequately administered to chimpanzees. However, given that bonobos and chimpanzees underwent the same administration procedure and most of individuals showed certain changes in eye-movement behavior, we think this possibility unlikely. One potential explanation is that intranasal OT reached the central nervous system and thereby affected behavior, but did not necessarily spread to the peripheral system or spread too little to be captured in our measurement system with chimpanzees. Further studies are necessary to test this idea. It should be noted that in previous studies which measured urinary OT after administration of OT in dogs (Nagasawa et al., 2015; Romero et al., 2014), administered OT led to certain behavioral changes as well as increase in urinary OT, but it is not clear whether the original (administered) OT reached urine; in fact the increase was not found in the absence of social interaction after OT/saline administration (Nagasawa et al., 2015). In previous studies which measured urinary OT in chimpanzees (Crockford et al., 2013; Samuni et al., 2017; Wittig et al., 2014), social interaction led to increase in urinary OT. There are mixed evidences showing that intranasally-administered OT reach to the peripheral system (i.e. blood) in macaques (Bauman et al., 2018). One study with capuchin monkeys showed an increase in urinary OT after intranasal administration of OT (Benítez et al., 2018), but unfortunately, an absence of saline placebo condition made an interpretation of this result somewhat difficult. Critically, no study has confirmed that intranasally administered OT per se reaches urine without behavioral mediations in apes, which we suspect unlikely following our preliminary results described below. Here, we submit these results as supplemental materials to help design future work.

\section{Methods:}

Sample collection. We conducted three tests using the same procedures expect the timings of urine sampling (Test 1-3). Six chimpanzees who also participated in our main behavioral study participated 
in this series of tests. One chimpanzee (Zamba) did not participate in Test 1 but all six chimpanzees participated in Test 2-3. All chimpanzees had previous experience urinating for sample collection, so urine could be directly caught in a cup while chimpanzees were in a corridor overhead. We also tested bonobos but decided not to use their urinary samples because we collected their urines from the floor (as they did not have previous experience urinating for sample collection); we then found that there were substantial variations in OT and creatinine levels, most likely due to contamination of water and other substances from the floor.

Upon collection of urine samples, urine was pipetted into test tubes, and placed on dry ice in a Styrofoam box exactly 5 minutes after urination. Notes were taken regarding unique characteristics of the samples, including color and volume. Immediately after completion of all samplings in the daily test, the urine samples were brought to a deep freezer and frozen at $-80{ }^{\circ} \mathrm{C}$ until shipment to a facility for analysis (during shipment, the samples were stored with dry ice in a Styrofoam box and placed in a freezer, $\left.-15^{\circ} \mathrm{C}\right)$.

In all tests, prior to administration of either OT or saline, chimpanzees were brought to the overhead corridor and urine was caught in a plastic cup (baseline). Immediately following this baseline urine collection, chimpanzees were given either oxytocin or saline placebo control using the same methods as in the behavioral tests (up to 10 minutes). After a wait period, which differed between the tests (90 minutes in Test 1 and 15 minutes in Test 2-3), chimpanzees were again moved to the overhead corridor for post administration urine collection. Five chimpanzees provided one urine sample between 90-120 minutes following the completion of administration procedure in Test 1 , and 6 chimpanzees provide multiple urine samples between 15-90 minutes and between 15-60 minutes following the completion of administration procedure in Test 2 and 3, respectively. The time period between 15-60 minutes in Test 3 was chosen to be consistent with the previous studies (Crockford et al., 2013; Samuni et al., 2017; Wittig et al., 2014). On a given day, half of subjects received oxytocin and half received saline, which was then counterbalanced on the next day of administration (minimum of 1 week later). Food and water intake were not restricted but carefully monitored. Chimpanzees were allowed to leave the corridor if they displayed any signs of discomfort, nervousness, or showed any attempt to leave, 
although the most participants provided at least one sample before their leave in all tests.

Urine analysis. Urinary oxytocin was measured using commercially available competitive ELISA (Enzyme-Linked Immunosorbent Assay) kit (ENZO Life Science, NY, UASA) either at a lab in Azabu University (Test 1-2) or its collaborator lab in Tokyo University of Agriculture (Test 3; AIRPLANTS BIO, Tokyo, Japan). Samples were centrifuged to remove dusts/tissues and then diluted by a factor of five. Sample extraction and concentration procedures were conducted following the official kit manual. Following incubation and washing (following kit instructions) results were calculated using a microplate reader $405 \mathrm{~nm}$. Final OT concentrations were calculated as pg OT per mg creatinine to control for variation in urine density.

Statistical analysis. As a dependent variable, we calculated the difference OT/cre score; we first divided the measured OT level $(\mathrm{pg} / \mathrm{ml})$ by the creatine level $(\mathrm{mg} / \mathrm{ml})$ for all samples, and subtracted the post-administration score from the baseline score. In Test 1, we compared between conditions using a paired t-test (one sample was collected from each participant in the post-administration period). In Test 2-3, we ran LMM in 'Ime4' with Gaussian error structure and identity link function. We included condition and time of urination (since the completion of the administration procedure; this variable was standardized), and their interaction as test predictors. The model checks were conducted as described in the main text. We included participants as a random intercept. Random slopes were kept maximal. The final model was: post OT/cre $\sim$ Condition*Time of urination $+(1+$ Condition*Time of urination | Subject). We used a likelihood ratio test to examine the significance of the terms. We dropped the interaction term if not significant, and then reran the model without the interaction term to examine the main effect of each term.

Results:

In Test 1 , the difference OT/cre score did not differ between OT and saline conditions $(\mathrm{t}(4)=0.19, \mathrm{p}$ $=0.86)$. In Test 2 , no effect was significant; the interaction effect $\left(\beta=-17.5, \mathrm{SE}=16.7, \mathrm{CI}_{\text {lower }}=-55.1\right.$, $\left.\mathrm{CI}_{\text {upper }}=18.9, \chi^{2}=0.94, \mathrm{p}=0.33\right)$; condition $\left(\beta=-34.6, \mathrm{SE}=29.3, \mathrm{CI}_{\text {lower }}=-99.3, \mathrm{CI}_{\text {upper }}=31.7, \chi^{2}=\right.$ $1.5, \mathrm{p}=0.23)$; time of urination $\left(\beta=-13.4, \mathrm{SE}=7.7, \mathrm{CI}_{\text {lower }}=-35.2, \mathrm{CI}_{\text {upper }}=5.7, \chi^{2}=1.5, \mathrm{p}=0.23\right)$. 
In Test 3, no effect was significant; the interaction effect $\left(\beta=-109.3, \mathrm{SE}=64.6, \mathrm{CI}_{\text {lower }}=-244.1, \mathrm{CI}_{\text {upper }}\right.$ $\left.=28.9, \chi^{2}=2.3, \mathrm{p}=0.13\right)$; condition $\left(\beta=30.6, \mathrm{SE}=64.5, \mathrm{CI}_{\text {lower }}=-109.6, \mathrm{CI}_{\text {upper }}=176.0, \chi^{2}=0.2, \mathrm{p}\right.$ $=0.68)$; time of urination $\left(\beta=37.0, \mathrm{SE}=22.8, \mathrm{CI}_{\text {lower }}=-11.2, \mathrm{CI}_{\text {upper }}=97.2, \chi^{2}=1.9, \mathrm{p}=0.17\right)$. Combining the results from Test 2 and 3 revealed a marginally significant interaction effect between condition and time of urination $\left(\beta=-91.6, \mathrm{SE}=46.6, \mathrm{CI}_{\text {lower }}=-187.1, \mathrm{CI}_{\text {upper }}=-7.4, \chi^{2}=3.4, \mathrm{p}=\right.$ 0.065). Accidentally, we detected a larger variation and generally higher values in urinary OT (but not in urinary creatine) in Test 3 compared to Test 1-2. We initially suspected minor technical differences in urinary OT analysis caused such differences because the Test-3 samples were analyzed in a different lab (due to the COVID-19 influence). We thus reanalyzed the Test-3 samples in the same lab where the Test-1-2 samples were analyzed (after the settlement), but confirmed similar OT values. Thus, the change in variation in OT level may be attributed to some other factors that we could not controlled for (e.g. seasonal change). Despite variation in absolute values, in theory, the difference between the conditions (OT/saline) should remain the same.
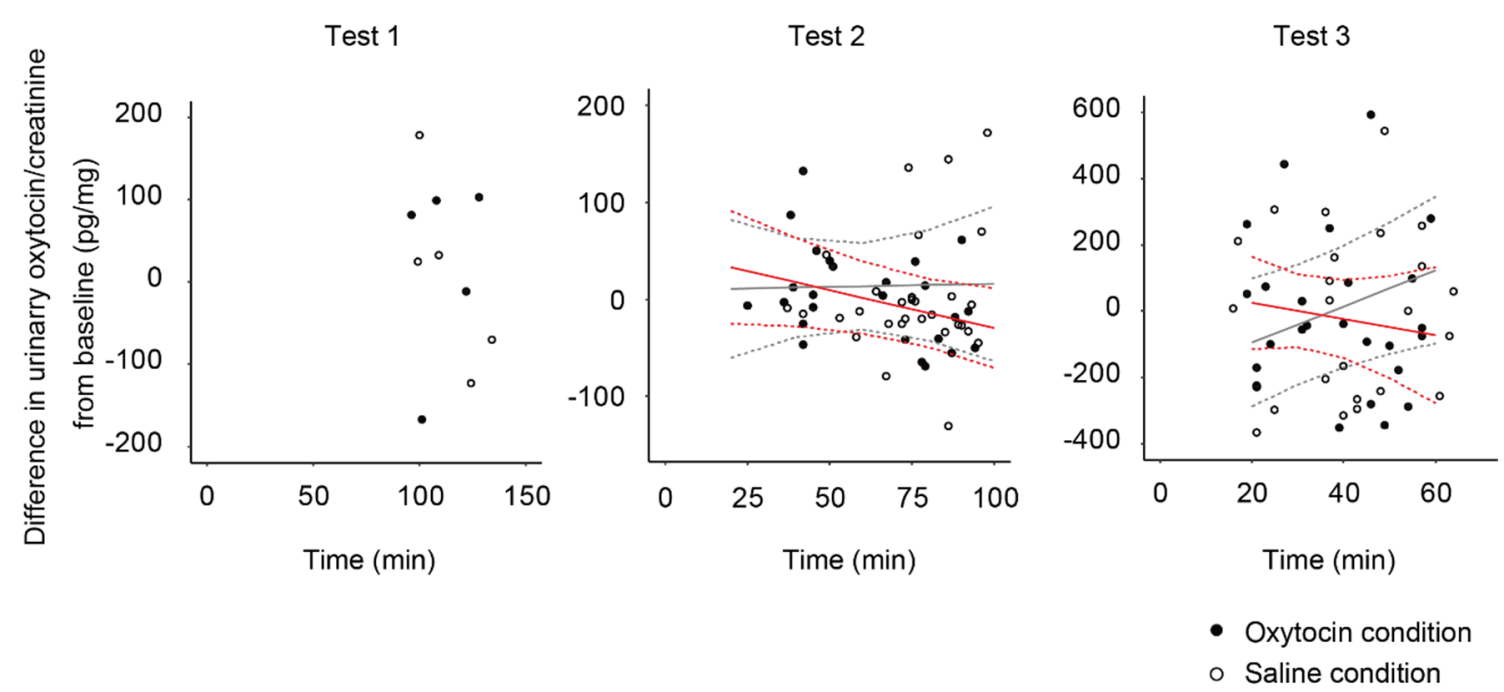

Figure S4. Difference in urinary oxytocin level $(\mathrm{pg} / \mathrm{ml})$ corrected for urinary creatine level $(\mathrm{mg} / \mathrm{ml})$ from baseline (pre-samples collected just before OT/saline administration). Solid and dotted lines indicate fitted values and confidence intervals, respectively (OT: red, Saline: gray). Regression lines and confidence intervals were drawn on the population level (without random-effects structure). 
References cited in Supporting Material

Bauman, M.D., Murai, T., Hogrefe, C.E., Platt, M.L., 2018. Opportunities and challenges for intranasal oxytocin treatment studies in nonhuman primates. Am. J. Primatol. 80, e22913-e22913.

Benítez, M.E., Sosnowski, M.J., Tomeo, O.B., Brosnan, S.F., 2018. Urinary oxytocin in capuchin monkeys: Validation and the influence of social behavior. Am. J. Primatol. 80, e22877.

Crockford, C., Wittig, R.M., Langergraber, K., Ziegler, T.E., Zuberbühler, K., Deschner, T., 2013. Urinary oxytocin and social bonding in related and unrelated wild chimpanzees. Proc. Roy. Soc. B 280, 20122765.

Kano, F., Hirata, S., Call, J., 2015. Social Attention in the two species of Pan: Bonobos make more eye contact than chimpanzees. PLoS One 10, e0129684.

Kano, F., Shepherd, S.V., Hirata, S., Call, J., 2018. Primate social attention: Species differences and effects of individual experience in humans, great apes, and macaques. PLOS ONE 13, e0193283.

Nagasawa, M., Mitsui, S., En, S., Ohtani, N., Ohta, M., Sakuma, Y., Onaka, T., Mogi, K., Kikusui, T., 2015. Oxytocin-gaze positive loop and the coevolution of human-dog bonds. Science 348, $333-336$.

Romero, T., Nagasawa, M., Mogi, K., Hasegawa, T., Kikusui, T., 2014. Oxytocin promotes social bonding in dogs. Proc. Nat. Acad. Sci. 111, 9085-9090. 
Samuni, L., Preis, A., Mundry, R., Deschner, T., Crockford, C., Wittig, R.M., 2017. Oxytocin reactivity during intergroup conflict in wild chimpanzees. Proc. Nat. Acad. Sci. 114, 268-273.

Wittig, R.M., Crockford, C., Deschner, T., Langergraber, K.E., Ziegler, T.E., Zuberbuhler, K., 2014. Food sharing is linked to urinary oxytocin levels and bonding in related and unrelated wild chimpanzees. Proc Biol Sci 281, 20133096. 